\title{
Integrated Genomics Analysis Identifies Recessive Ciliopathy Mutations in Primary Endocardial Fibroelastosis: a Rare Neonatal Cardiomyopathy
}

\section{Yan Zhao}

University of California Los Angeles

\section{Wang Lee-Kai}

University of California Los Angeles

\section{Ascia Eskin}

University of California Los Angeles

Xuedong Kang

University of California Los Angeles

Viviana M. Fajardo

University of California Los Angeles

\section{Zubin Mehta}

UCLA

\section{Stacy Pineles}

University of California Los Angeles

Ryan J. Schmidt

University of Southern California

\section{Aaron Nagiel}

University of Southern California

\section{Gary Satou}

University of California Los Angeles

\section{Meena Garg}

University of California Los Angeles

Myke Federman

University of California Los Angeles

\section{Leigh C. Reardon}

University of California Los Angeles

Steven L. Lee

University of California Los Angeles

\section{Reshma Biniwale}

University of California Los Angeles

Wayne W. Grody 
University of California Los Angeles

\section{Nancy Halnon}

University of California Los Angeles

\section{Negar Khanlou}

University of California Los Angeles

\section{Fabiola Quintero-Rivera}

University of California Irvine

\section{Juan C. Alejos}

University of California Los Angeles

\section{Atsushi Nakano}

University of California Los Angeles

\section{Gregory A. Fishbein}

ucla

\section{Glen S. Van Arsdell}

University of California Los Angeles

\section{Stanley F. Nelson}

University of California Los Angeles

Marlin Touma ( $\nabla$ mtouma@mednet.ucla.edu )

University of California Los Angeles https://orcid.org/0000-0002-2827-4068

\section{Research Article}

Keywords: Primary Endocardial Fibroelastosis, Neonatal Cardiomyopathy, Alstrom Syndrome, Exome Sequencing; RNA sequencing; Fibrosis; Epithelial Mesenchymal Transition, Retinal Dystrophy, Rare Undiagnosed Disease

Posted Date: March 26th, 2021

DOI: https://doi.org/10.21203/rs.3.rs-327918/v1

License: (c) (1) This work is licensed under a Creative Commons Attribution 4.0 International License. Read Full License

Version of Record: A version of this preprint was published at Journal of Molecular Medicine on August 13th, 2021. See the published version at https://doi.org/10.1007/s00109-021-02112-z. 


\section{Abstract}

Among neonatal cardiomyopathies, primary endocardial fibroelastosis ( $\mathrm{pEFE}$ ) remains a mysterious disease of the endomyocardium, affecting $1 / 5000$ live births and accounting for $25 \%$ of the entire pediatric dilated cardiomyopathy with a devastating course and grave prognosis. To investigate the potential genetic cause that underlies $\mathrm{pEFE}$, we performed integrative genomic analysis, using whole exome sequencing (WES) and RNA-seq in a female infant with confirmed pathological diagnosis of pEFE. Within regions of homozygosity in the proband genome, WES analysis revealed novel parent-transmitted homozygous mutations affecting three genes with known roles in cilia assembly or function. Among them, a novel homozygous variant [c.1938delA] of uncertain significance in ALMS1 was prioritized for functional genomic and mechanistic analysis. Loss of function mutations of ALMS1 have been implicated in Alstrom syndrome (AS) [OMIM 203800], a rare recessive ciliopathy that has been associated with cardiomyopathy. The variant of interest results in a frameshift introducing a premature stop codon. RNA-seq of the proband's dermal fibroblasts confirmed the impact of novel ALMS1 variant on RNA-seq reads and revealed dysregulated cellular signaling and function, including the induction of epithelial mesenchymal transition (EMT) and activation of TGF $\beta$ signaling. ALMS1 loss enhanced cellular migration in patient fibroblasts as well as neonatal cardiac fibroblasts, while ALMS1-depleted cardiomyocytes exhibited enhanced proliferation activity. Herein we present the unique pathological features of pEFE compared to DCM and utilize integrated genomic analysis to elucidate the molecular impact of a novel mutation in ALMS1 gene in an AS case. Our report provides insights into pEFE etiology and suggests, for the first time to our knowledge, ciliopathy as a potential underlying mechanism for this poorly understood and incurable form of neonatal cardiomyopathy.

\section{Background}

Neonatal cardiomyopathies are multifactorial disorders that manifest early after birth as an isolated trait or as a part of a syndromic picture [1]. Syndromic cardiomyopathies are characterized by severe, earlyonset ventricular dysfunction accompanied by multisystem disorders. Remarkably, the features of different syndromes may overlap $[1,2]$. Hence, the complexity and rarity of these disorders, together with delayed systemic manifestations, different expressivity, and early lethality, make accurate and timely diagnosis difficult. Although we do not fully understand the underlying etiology of these rare and serious disorders, genetic defects play vital roles and may converge on common molecular pathways to different degrees. Next-generation sequencing of DNA and RNA have proved efficient for the early detection of cardiomyopathies allowing the discovery of many genes associated with isolated or syndromic forms, paving the way for elucidating the disease mechanism and identifying disease-specific therapy $[3,4]$.

Primary endocardial fibroelastosis ( $\mathrm{pEFE}$ ) is a rare form of neonatal cardiomyopathy that occurs in $1 / 5000$ live births with significant consequences but unknown etiology [5-9]. It is one of the most challenging diseases of the endomyocardium, in which the deposition of subendocardial fibrous tissue during early postnatal heart development leads to significant thickening of the endocardium and restriction of left ventricular filling and growth [5]. Patients with pEFE present during early infancy with 
progressive left ventricular (LV) dysfunction leading to congestive heart failure and early death in $80 \%$ of cases. A published report from the UCLA-pediatric heart transplant program indicates that pEFE accounts for approximately $25 \%$ of the entire pediatric dilated cardiomyopathy (DCM) cohort, while comprising $80 \%$ of cardiac transplantation undertaken for patients with DCM before one-year of age [5]. In contrast to secondary EFE that occurs in association with structural heart malformation such as hypoplastic left ventricle, the anatomy of pEFE hearts is generally normal [5]. Compared to other DCM subtypes, the phenotypic features of pEFE are unique and consistent [5]. The LV is usually dilated with extensive thickening of the endocardium by diffuse elastin and collagen fibers that limit ventricular diastolic compliance. The thickening further extends to the mitral valve and chordae tendineae leading to upward displacement of the papillary muscles and mitral regurgitation. Histologically, sub-endocardial vacuolization of the myocytes with perinuclear clearing has been described [5]. At the ultrastructural level, myofibrillar disarray, widening Z-bands, disorganized intercalated disks, myofibrils' loss, perinuclear glycogen deposits, and variation in mitochondrial morphology were observed in electron micrographs [9]. Despite being recognized as a distinct pathological entity, the diagnosis of pEFE is entirely based on postmortem or post-transplant, pathological examination of the diseased heart.

The genetic model of pEFE remains unresolved. Familial aggregation of $p E F E$ has been reported ranging between $8 \%$ and $18 \%$; however, remarkable genetic heterogeneity exists [10-11]. Mutations of the TAZ gene, which encodes a mitochondrial targeted acyltransferase known as tafazzin, have been implicated in an X-linked form of pEFE in association with features of BARTH syndrome [OMIM 300394] [11]. However, the genetic mechanism and the mode of inheritance remain unknown in the vast majority of pEFE cases where sporadic isolated cardiomyopathy is presented with negative family history. Since cardiac transplantation is becoming more feasible for infants with end-stage heart failure, establishing an early diagnosis is essential to clinicians in making decisions regarding transplant candidacy. Moreover, elucidating the disease mechanism is necessary to identify targeted therapies. However, this task has been challenging due to the multifaceted complexity of myocardial disease in general, and particularly due to the critical gaps in our knowledge of this disease entity and the limited success in disease modeling necessary for in-depth mechanistic interrogation.

Alstrom syndrome (AS) [OMIM: 203800] is an extremely rare, autosomal recessive, Mendelian disorder with a prevalence of less than 1 per million in the general population [12-20]. As a ciliopathy disoreder, AS typically manifests during infancy or childhood, although some features may occur later in life. Since cilia are expressed ubiquitously and are involved in various signal transduction pathways, AS is typically associated with severe multi-organ defects. Patients develop progressive cone-rod dystrophy leading to blindness, neuronal hearing loss, early onset truncal obesity, hypertriglyceridemia, insulin resistance, hyperinsulinemia, type 2 diabetes, short stature, renal failure and liver dysfunction. Patients are also susceptible to multi-organ failure secondary to widespread fibrosis of unknown etiology. Reportedly, cardiomyopathy is an important feature of AS and may manifest during infancy, as a mitogenic cardiomyopathy [16-18], or later in childhood as dilated cardiomyopathy $[19,20]$. However, the pEFE phenotype was never described in association with AS. 
Aiming to elucidate the genetic cause of pEFE, we carried out an integrative genomic analysis, using trio whole-exome sequencing (WES) and RNA-seq, on a female proband with confirmed diagnosis of pEFE and her unaffected parents. We identified a novel homozygous single nucleotide deletion, which results in a frameshift [p.Val649fs] and introduction of a premature stop codon in $A L M S 1$, suggesting the genetic diagnosis of AS $[12,13]$. Clinical follow-up and integrative genomic analysis of WES and RNA-seq of the proband-derived fibroblasts confirmed the causality of the novel ALMS1 variant and elucidated the molecular signature of pEFE learned from our index AS case. To our knowledge, our report provides the first evidence linking ciliopathy to pEFE etiology.

\section{Results}

Human Studies: All human studies were conducted in accordance with regulation of the University of California Los Angeles (UCLA) Institutional Review Board (IRB). Subjects provided written informed consent to participate in this study. Electronic medical record, family pedigree and specimen collection were acquired through the UCLA Congenital Heart Defect (CHD) BioCore [21] following the UCLA-IRB approved protocols. Specimens were de-identified and coded following acquisition.

Case of Interest: The pEFE proband is a female infant of healthy parents of Hispanic descent, who presented with profound congestive heart failure at 6 weeks of life. The prenatal course and delivery history were uneventful. Newborn screening, infectious work-up and comprehensive metabolic panel revealed no abnormalities [Figure 1. A and B]. pEFE diagnosis was determined based on clinical pathological examination of the explanted heart. No structural defect was identified. At the time of heart transplantation, the ejection fraction EF of the left ventricle was less than $20 \%$. The clinical time course of the participant is summarized in Figure 1. C.

Pathological Findings: Pathological examination was performed by an expert anatomic pathologist. A gross image of the explanted pEFE heart is shown in Figure 1. D. Diffuse thickening of LV endocardium with an elevation of the papillary muscles and thickening of the free edges of mitral valve leaflets were observed, giving an opaque appearance to the endocardial surface. Compared to an age matched heart with dilated cardiomyopathy (DCM), the LV endocardial thickness is significantly greater in pEFE [Figure 1.E a\&b] with dense elastic fibers arranged in a longitudinal pattern shown by Trichrome staining [Figure 1.E c\&d]. In contrast, collagen IV deposits were less pronounced [Figure 1.E e\&f]. Based on these pathological features, a clear distinction could be made between pEFE heart and DCM heart.

Potential Contribution of Recessive Ciliopathy Genes to pEFE Etiology:To investigate the genetic contribution to pEFE etiology, we performed WES on our pEFE proband and her parents combined with RNA-seq analysis of pEFE proband dermal fibroblasts [Supplemental Figure 1]. Parents received pre-test genetic counseling and were then asked to provide informed consent to make anonymous clinical and genomic data available for research and publication. Using a custom-made primary gene list of 44 known cardiomyopathy genes, including TAZ [Supplenmental Table 1], no pathogeneic variants were identified. Based on the sporadic nature of pEFE presentation in the proband cohort, we expected that de novo 
germline mutations might contribute to the genetic basis of pEFE. However, no denovo mutation was detected. The family pedigree did not reveal parental consanguinity or another affected family member. On the contrary, homozygosity analysis revealed 8 large runs of homozygosity (ROH) greater than $5 \mathrm{MBs}$, totaling $51.8 \mathrm{MBs}$, which correspond to $1.62 \%$ of the proband's genome [Supplemental Table 2], suggesting that both parents descend from a common, genetically isolated community. Importantly, within these ROHs, three novel homozygous variants in cilia-related genes, DNAH8, DNAH17, and ALMS1, were detected, while both of the unaffected parents were heterozygous carriers for each of these variants [Figure 2. A]. Integrative Genomics Viewer (IGV) analysis confirmed the zygosity of the variants [Figure 2. B]. The detected variants have not been previously reported in gnomAD, ClinVar (https://www.ncbi.nlm.nih.gov/clinvar/), the 1000 Genomes database (http://www.internationalgenome.org), or ExAC (http://exac.broadinstitute.org/). By Sanger sequencing, we confirmed the newly identified homozygous variants using gDNA obtained from dermal fibroblasts derived from a punch skin biopsy of the pEFE proband, with human neonatal dermal fibroblasts (hNDFs) used as a negative control [Figure 2.C].

DNAH8 and DNAH17 genes encode two heavy chain proteins of the axonemal dynein that generate force through ATP hydrolyzing and microtubule binding. The novel homozygous variant in DNAH17 [17:76487553 c.6641C>G] replaces a serine residue for cysteine at the amino acid 2214 (p.Ser2214Cys) and is predicted to be deleterious, suggesting this variant may not be well tolerated. The novel homozygous single nucleotide deletion in DNAH8 [6:38840474 c.7153delT] results in a frame shift [p.Phe2385fs] that causes a premature termination of translation leading to a truncated protein that only contains the first 2384 amino acids of DNAH8 followed by an extra stretch of 23 amino acids. Therefore, the ATPase activity and microtubule-binding ability are predicted to be affected by the mutation. Variants in DNAH17 and DNAH8 have been implicated in spermatogenic failure [OMIM: 618643] [22], and have never been reported in association with cardiomyopathy. Therefore, these two genes are unlikely to contribute to pEFE phenotype.

Finally, ALMS1 encodes the centrosome and basal body associated protein 1, which functions in the formation and maintenance of cilia and in microtubule organization [12, 13]. The novel ALMS1 variant [2: 73675594 c.1938delA] is a single nucleotide deletion, which results in a frameshift [p.Val649fs] and introduction of a premature stop codon. Mutations in ALMS1 are known causal of AS [OMIM 203800] [1215], a rare autosomal recessive ciliopathy, which has been associate with cardiomyopathy [16-20]. However, AS has not been associated with pEFE phenotype and our patient did not have other clinical features of AS at the time of WES studies.

Prioritizing the Candidate Genes for Functional Assessment: To further prioriterize the candidate novel variants in the three cilia-related genes, we set out first to confirm the impact of these variants on RNA expression. We isolated total RNAs from pEFE proband dermal fibroblasts and from hNDFs (as a control) and performed paired-end RNA-seq as we previously described [23, 24]. Out of the three candidate genes, ALMS1 was the only gene expressed in dermal fibroblasts with good read coverage as illustrated by IGV viewer, while neither DNAH8 nor DNAH17 was detected. IGV confirmed the impact of the novel ALMS1 
c.1938delA variant at the RNA level in pEFE fibroblast compared to control hNDF [Figure 2. D]. Next, we examined the expression of these genes in our previously reported RNA-seq data of congenital heart defects samples obtained from infants with structural heart defects [24]. Among the three genes, only ALMS1 was expressed in heart tissue samples [Figure 2. E].

Given that the cardiac dysfunction of pEFE manifests clinically during the early postnatal period [5], we next examined the expression of the three candidate genes in our previously reported RNA-seq data derived from neonatal mouse heart left and right ventricles at postnatal day P0, P3 and P7 [24], available in the NCBI's Gene Expression Omnibus repository under the Neonatal Heart Maturation SuperSeries [http://www.ncbi.nlm.nih.gov/geo/query/acc.cgi?acc=GSE85728]. Again, among the three candidate genes, ALMS1 was the only gene expressed in both ventricular chambers, exhibiting dynamic regulation in neonatal heart during perinatal stages [Figure 2. F]. To further ascertain the expression pattern of the candidate genes in neonatal heart at the cellular level, we examined their expression in primary cultured neonatal rat ventricular myocytes (NRVMs) and neonatal rat cardiac fibroblasts (NRCFs) isolated from neonatal rat hearts. Indeed, out of the three candidate genes, only ALMS1 was expressed in both cardiac cell types [Figure 2. G]. Altogether, our findings led to prioritizing ALMS1, which is expressed in neonatal heart and has been associated with cardiomyopathy, as the potential causal candidate gene contributing to $\mathrm{pEFE}$. Therefore, we focused the following studies on determining the molecular and cellular impact of the novel ALMS1 variant in pEFE pathogenesis.

ALMS1 Protein Expression is Absent in pEFE Proband Dermal Fibroblasts: ALMS1 gene is located on chromosome 2p13.1 spanning 23 exons. It encodes a protein of 4,169 amino acids ( $461.2 \mathrm{kDa}$ ), which lacks known catalytic domains $[25,26]$, but has several sequence features of unknown function, including a large tandem repeat domain (TRD), three short coiled-coil domains and a stretch of $\sim 130$ residues at the C-terminus termed as the ALMS motif [aa: 4032-4164 (Ensembl)] [Figure 3. A]. The ALMS1 protein is widely expressed and cotocalizes with the centrosomes and basal bodies of ciliated cells in different tissues and organs, where ALMS1 has been shown to play important roles in ciliary function and intracellular trafficking [25]. Deletion analysis of ALMS1 suggests that the ALMS motif may play a key role in centrosome-targeting [26]. Importantly, the variant of interest [c.1938delA] is predicted to result in a severely truncated ALMS1 protein [p.Val649fs], missing most of the tandem repeats and the highly conserved ALMS motif at the C-terminus. Based on these previously reported observations, we predicted the ALMS1 protein expression and/or stability in the centrioles to be affected by the novel ALMS1 variant in pEFE cells. Indeed, immunocytochemistry (ICC) assay using anti-ALMS1 antibodies confirmed ALMS1 protein localization to the centrioles in control hNDFs, while ALMS1 protein was completely absent in the proband pEFE-derived dermal fibroblasts, indicating a null effect [Figure 3. B].

Novel ALMS1 Variant Alters the Functional Phenotype of pEFE Fibroblasts: To examine the functional impact of the novel ALMS1 variant at the cellular level, we first examined cilia morphology in pEFE dermal fibroblasts compared to control hNDFs using electron microscopy (EM). We observed a unipolar organization of thin, over-branched, microvilli, nonmotile finger-like protrusions from the surface of epithelial cells that function to increase the adhesion, the cell surface area, the efficiency of absorption, 
that was more prominent in pEFE cells compared to control hNDFs [Figure 3. C]. Next we examined the impact on cellular proliferation over time using an automated proliferation assay performed on an xCELLigence RTCA SP instrument. We observed decreased proliferation index in pEFE fibroblasts, compared to hNDFs [Figure 3. Dand E]. FACS analysis revealed no change in G2/M phase in pEFE fibroblast compared to control [Supplemental Figure 2]. Then, we examined the impact on cellular migration over time using the xCELLigence RTCA DP instrument and observed increased migration activity in pEFE fibroblasts compared to the control hNDFs [Figure 3. F and G]. Together, the novel ALMS1 variant altered the functional phenotype and cellular physiology of pEFE fibroblasts. To further confirm the impact of ALMS1 loss, we treated primary cultured hNDF1 with ALMS1-siRNA. Indeed, ALMS1depleted hNDFs exhibited enhanced migration replicating the changes seen in pEFE fibroblasts [Supplemental Figure 3].

Novel ALMS1 Variant Impact on pEFE Proband Heart: As previously stated, few reports have implicated homozygous ALMS1 mutations in an extremely rare form of neonatal cardiomyopathy associated with AS and characterized by prolonged mitotic window of postnatal cardiomyocytes, hence termed "mitogenic cardiomyopathy" [16, 17]. Furthermore, mice with Alms1 mutations exhibit delayed exit from active cell cycle progression [18], supporting a functional role for ALMS1 in regulating postnatal cardiomyocyte maturation. Based on these reported observations, we sought to determine the pathogenic impact of the mutant ALMS1 in pEFE heart. First, we determined that ALMS1 protein is also localized at the centrosomic poles (centrioles) in neonatal cardiomyocytes by using primary cultured neonatal rat ventricular myocytes (NRVMs) isolated from the neonatal rat heart [Figure 4. A]. Next, we confirmed that ALMS1 loss is sufficient to extend the proliferative activity of postnatal cardiomyocytes by using small interfering RNA (siRNA)-mediated inhibition of $A / m s 1$ in primary cultured NRVMs. We observed increased mitotic activity in Alms1-deficient myocytes, as indicated by increased number of phospho-Histone $\mathrm{H} 3$ $(\mathrm{pH} 3)$ positive cardiomyocytes and upregulation of the mitotic marker genes, including Ki67 and Cdc25c, compared to control scramble [Figure 4. B and C]. These results are consistent with previous studies [1618], indicating that ALMS1 regulates neonatal cardiomyocyte proliferation. Then, we confirmed that ALMS1 expression at the centrosomes was absent in histopathological sections obtained from the proband pEFE heart, but appeared normal in the age matched DCM heart with a pathogenic mutation in TNNT2 [Figure 4. D]. Finally, we evaluated cardiomyocyte proliferation activity in proband pEFE heart sections, using $\mathrm{pH} 3$ immunohistochemistry $(\mathrm{IHC})$ analysis and revealed increased $\mathrm{pH} 3$ positive cardiomyocytes in PEFE heart compared to the age-matched DCM heart [Figure 4. E and F]. Together, in agreement with other reported cases of AS-associated mitogenic cardiomyopathy, the novel ALMS1 variant abolished ALMS1 protein expression and localization at the centrioles and delayed cardiomyocyte proliferation arrest in pEFE heart. However, how novel ALMS1 vaiant leads to pEFE phenotype remains unclear.

Novel ALMS1 Variant Alters Global Transcriptome Signature in pEFE Fibroblasts: Having established the causal role of the novel ALMS1 variant [c.1938delA] in pEFE, we aimed to gain further insights into the mechanisms that underlie ALMS1 function in endocardial fibroelastosis process by evaluating the global impact of ALMS1 loss on transcriptome programming of proband pEFE cells that carry the mutation. We 
systematically analyzed pEFE dermal fibroblast-derived RNA-seq datasets using total RNA obtained from three independent biological replicates. In addition, three independent hNDF replicates were subjected to the same RNA-seq protocol as we previously described $[23,24]$.

Principal component analyses of the top 1000 varied genes showed that transcripts from pEFE and control fibroblasts formed distinct clusters [Figure 5. A]. Likewise, expression heatmap across the entire transcriptome revealed distinct molecular signatures for pEFE and control samples [Figure 5. B], indicating that the variation pattern was consistent across the two methods. In total, 8970 protein coding genes were expressed at $\geq 3$ RPKM in at least three sample (3 biological replicates) with coefficient of variation (CV) exceeding 0.2. Of these expressed genes, 3910 genes exhibited significant differential gene expression (DGE) in pEFE versus control at Benjamini-Hochberg (B-H) adjusted $P$ value less than 0.05 [Figure 5. C]. Together, these findings indicate significant impact of $A L M S 1$ perturbation on global transcriptome signature in pEFE fibroblasts compared to the control hNDF cells.

\section{Novel ALMS1 Variant Induces TGFß Signaling and Activates Epithelial Mesenchymal Transition (EMT):}

Gene ontology analysis of the differentially expressed genes was performed using Gene Set Enrichment Analysis (GSEA) [27]. EMT, a process that converts epithelial cells to mobile mesenchymal cells and plays an important role in cardiac development [28], was predominantly enriched in the upregulated genes. Altogether, 175 EMT-related genes exhibited significant induction [Supplemental Tables 3-5], including activated myofibroblast cell surface marker genes (EDA, POSTN), cell adhesion genes (ITGB3, ITGA2), extracellular matrix (ECM) genes (COL5A3, ELM), key transcription regulators of EMT (ID1, ID2, SNAIL), and major signaling players of EMT $(F Z D 8, T G F \beta)$. Importantly, consistent with acquiring enhanced motility and migration abilities, genes involved in ECM degradation (MMP14, LOXL1) and motility (FGFR2, PRKG2) were also upregulated. On the other hand, apical junction (JUP, CDSN), cytoskeletal organization (NEXN, ABLIM1), P53 signaling (PIDD1, CASP1), intercellular trafficking (SLC16A6, SLC29A2) and cell cycle genes were downregulated in pEFE4 fibroblast compared to control. Together, the molecular signature of pEFE fibroblasts is consistent with a migratory, non-proliferative, EMT induced cellular phenotype.

To further annotate the enriched functional identity and signaling pathways in the differentially expressed genes, we used Kyoto Encyclopedia of Genes and Genomes (KEGG) pathway enrichment analysis and identified cellular adhesion, exon guidance, TGF $\beta$ and RAP1 signaling as the top enriched functional pathways that were also interconnected by sharing several overlapping genes [Figure 5. D and E]. For example, genes involved in EMT significantly overlapped with cell adhesion molecule and TGF $\beta$ pathways. Among these hub genes, we identified BMPR1B, a known member of the bone morphogenetic protein (BMP) family of transmembrane serine/threonine kinases that has been associate with left ventricular mass [29]. The ligands of this receptor are members of the BMPs and TGF- $\beta$ superfamily. Consistently, upstream analysis using IPA (Ingenuity Pathway Analysis) of the connected networks predicted TGF $\beta$ as the top upstream regulator with remarkable induction of its downstream signaling mediators, including BMPR1B, ID1-4, SMAD9, INHBB, CHRD, and BMP6, many of which are key players of 
EMT during embryonic development and cardiogenesis [28] [Supplemental Tables 3-5]. Together, our findings suggested TGF $\beta$ mediated activation of EMT program in the proband $p E F E$ fibroblasts.

To validate these findings, we examined the impact of the novel ALMS1 variant on selected EMT marker genes in primary cultured pEFE fibroblasts compared to control hNDFs using qRT-PCR. Indeed, TGF $\beta$ was induced along with the transcription factor SNAIL, the ECM marker ELN as well as the myofibroblast marker gene aSMA. Consistently, $C D H 1$, a key signaling mediator for the induction of EMT cascade, was significantly upregulated, while $C D H 2$ was downregulated. Finally, cell cycle markers (Ki67 and PIk1) were suppressed [Figure 6. A]. Together, the data support that ALMS1 loss leads to EMT induction, potentially via the activation of TGF $\beta$ signaling.

Novel ALMS1 Mutation Induces EMT in Neonatal Cardiac Fibroblasts: Current evidence suggests a role for EMT in EFE [30, 31]. Our transcriptome analysis suggested that ALMS1 loss altered the physiological proprieties (enhanced migration) and induced EMT in pEFE fibroblasts potentially via activating TGF $\beta$ signaling. We sought next to determine whether ALMS1 regulates EMT in cardiac fibroblasts by performing siRNA-mediated knockdown of Alms1 in primary cultured NRCFs. Remarkably, the cells exhibited enhanced migration and induced EMT marker genes, including the Snail1, Tgfb, and Cdh1 [Figure 6. B-D]. Importantly, unlike neonatal cardiomyocytes, NRCFs remained quiescent and did not exhibit increased proliferation activity as demonstrated by proliferation marker genes (Plk1 and Ki67) expression [Figure 6. D]. Together, the data suggest EMT induction and Tgfb activation in ALMS-deficient neonatal cardiac fibroblasts. These findings replicate the changes observed in the proband pEFE dermal fibroblasts and ALMS1-deficient hNDFs, and correspond to a known role of cilia-mediated signaling in driving EMT process in cardiac development and fibrosis in response to cardiac injury [32].

Further Surveillance for Alstrom Syndrome and Patient Care: According to the diagnostic criteria from Marshall et al (2007) [13], detecting the novel ALMS1 variant [c.1938delA] suggested the genetic diagnosis of AS. We provided detailed genetic consultation about AS to the family and monitored our pEFE proband closely for potential multisystem involvement, in addition to the regular monitoring and management by the primary cardiologist and heart transplantation clinic. Bilateral nystagmus, ptosis and photophobia were documented at 6-12 month of age. Visual impairment was diagnosed at one year of age. Standard electroretinography (ERG) at 7 years of age showed absence of scotopic, photopic, maximal-combined and flicker responses, consistent with severe pan-retinal abnormalities of both rodand cone- mediated retinal functions [Figure 7. A]. Retinal imaging revealed bilateral retinal dystrophy [Figure 7. B]. Short stature, increased weight gain and early onset obesity were observed at 2-3 years of age associated with elevated triglycerids, acanthosis nigricans and increased hemoglobin A1C (HbA1C) suggesting insulin resistance [Figure 7. C-E]. Hearing evaluation, thyroid function tests, blood sugar levels, serum lipid profile, liver function tests, serum creatinine, abdominal ultrasound and blood chemistry remained normal. The patient maintained normal bilateral audiology and psychomotor functions.

\section{Discussion}


In this report, we describe the unique pathological finding of pEFE compared to DCM. We also elucidate novel genetic contribution to pEFE etiology involving a known ciliopathy gene. By complementing triobased WES with RNA-seq of patient-derived fibroblasts, our integrative genomic analysis allowed the identification of a novel pathogenic variant. Herein we report our novel discovery of ALMS1 as a causal gene underlying pEFE pathogenesis. To our knowledge, this is the first case of pEFE associated with Alstrom syndrome (AS), a rare recessive ciliopathy.

Our report expands the phenotypic and genetic features of pEFE and AS. The patient reported here initially presented with a unique form of isolated neonatal cardiomyopathy that could lead to early death [5]. Subsequent WES analysis revealed the genetic diagnosis of AS. Having survived heart transplantation at one year of age, surveillance for multi-organ involvement and targeted interventions can help improve long-term outcomes for this patient [19]. Since ALMS1 is the only gene in which recessive mutations are known to cause AS manifisatations, including cardiomyopathy [12-20], we ascertained the inheritance pattern, leading to appropriate counseling. Therefore, we propose utilizing WES as the first diagnostic tool to establish an early diagnosis for neonatal cardiomyopathies, ALMS1 should be added to the cardiomyopathy gene panel, the cardiomyopathy in ALMS1, in this case at least, is pEFE, and that patients with cardiomyopathy/pEFE should be investigated for syndromic features of Alstrom syndrome

The giant ALMS1 protein, predicted to comprise 4,169 amino acids, is widely expressed and localized to the centrosomes and basal bodies of ciliated cells of different organs $[\mathbf{2 5}, \mathbf{2 6}]$. ALMS1 loss-of-function has been linked to defects in primary cilium formation, positioning and maintenance, leading to AS being classified as a ciliopathy $[12,13]$. Reportedly, the ALMS motif is the only region that shares obvious sequence similarity with other human proteins and confers an evolutionally conserved function in targeting ALMS1 protein to the centrioles [26]. Consistent with previous reports, the ALMS1 protein was absent in the centrioles of the proband pEFE fibroblasts and heart tissue sections, confirming a deleterious effect of the novel ALMS1 variant on ALMS1 protein translation and localization. Although the exact functions of the ALMS1 protein are yet to be fully revealed, it has been demonstrated to play a role in cell cycle regulation [16-18]. Consistently, proliferation activity was enhanced in pEFE heart tissue compared to DCM. Likewise, Alms1-depleted cardiomyocytes exhibited a modest increase in their mitotic activity. On the contrary, Alms1-depleted NRCFs and dermal fibroblasts from pEFE proband exhibited reduced proliferative capacity. However, they demonstrated enhanced migration abilities when compared to their control counterparts. Together, these conflicting phenotypic properties in ALMS1-depleted fibroblasts, in contrast to cardiomyocytes, may suggest cell type-specific role for ALMS1 protein in heart and potentially other organs.

Alms1-deficient mice have been studied and the initial formation of primary cilia appeared normal in all three mouse models reported to date. However, age-dependent loss of primary cilia was observed in two

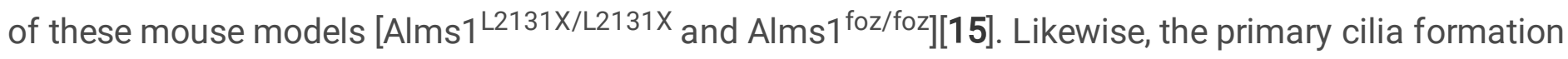
seems to be unaffected in reported AS patient fibroblasts in vitro [33]. Consistent with these reports, primary cilia formation appeared normal in our proband [pEFE] fibroblasts. The observed unipolar localization and confluent networking of branching cilia is potentially consistent, in part, with the 
observed enhanced cellular motility and migration properties. Whether these differences in the cilia-loss occurrence/timing, seen in previous murine studies, are mutation specific or cell type-specific remains to be interrogated.

Cilia play essential roles throughout cardiac development. During cardiogenesis, primary cilia are present on the majority of heart cell types acting as cellular sensors for signal transduction that control cardiac myocytes and fibroblast differentiation [34-36]. Deletions in cilia-related genes have been implicated in congenital heart defects such as heterotaxy, which may manifest clinically as an isolated disorder or associated with other features of situs inverses $[34,35]$. In the case of AS, dilated cardiomyopathy during childhood is a common feature $[19,20]$. Meanwhile, only few reports have described an extremely rare feature of AS called "mitogenic cardiomyopathy" that is characterized by persistent mitotic activity in neonatal cardiomyocytes [16-18]. Importantly, like pEFE, AS-associated mitogenic cardiomyopathy can be lethal during infancy, before other clinical features of AS normally manifest. However, in contrast to the unique pEFE phenotype, no abnormalities in the endocardium have been described in mitogenic cardiomyopathy cases that are reported thus far. Indeed, our report provides the first link between ciliopathy and pEFE.

How ALMS1 contributes to pEFE remains to be fully revealed. The involvement of the endocardium is a distinct, yet mysterious, phenomenon in pEFE hearts. Unlike secondary EFE that associates with hypoplastic left heart or myocarditis, no structural defect is seen in pEFE cases, meriting further investigations. Hence, unusual human phenotypes offer the opportunity to understand disease pathogenesis, if the cause can be determined. Having identified a causal homozygous ALMS1 variant in pEFE4, the availability of dermal fibroblasts derived from pEFE4 allowed us to examine the broader impact at the molecular and cellular levels by performing unbiased transcriptome analysis of the patient cells that harbor the ALMS1 [c.1938delA] mutation, using hNDFs as control. EFE is a specialized form of cardiac fibrosis that involves excessive fibroblast-mediated deposition of elastin (ELM) and collagen fibers, predominantly, in the subendocardium layer of the left ventricle [6-9]. Consistently, ALMS1-deficient fibroblasts from pEFE4 exhibited significant upregulation of several ECM constituents compared to hNDF [Supplemental Table 5].

Different cardiac lineages arise from one or more EMT waves during caridiogenesis. Errors of the EMT process, including ciliopathies, may lead to cardiac defects and fibrosis [28, 29]. An epithelial cell must disassemble the cell-cell junctions, lose epithelial cell polarity, and acquire a motile phenotype to undergo EMT. The Snail proteins are key EMT-inducing transcription factors activated by EMT-inducing stimuli such as TGF $\beta$. In turn, Snail suppresses adhesion protein genes such as E-cadherin and upregulates the cytoskeletal proteins leading to cytoskeletal reorganization. Furthermore, it stimulates the expression of matrix metalloproteases (MMPs) that break down the ECM and facilitate cellular migration. It was previously suggested that the EFE fibroblasts arise from postnatal endocardial cells that undergo an aberrant endothelial-to-mesenchymal transition (End-MT) [30]. However, using an EFE-like mouse model with hemodynamically unloaded heart and multiple genetic lineages tracing approach, a more recent study showed that the epicardium-derived mesenchymal cells (Epi-MCs) serve as the major 
source for EFE fibroblasts [31]. In agreement, pEFE fibroblast transcriptome profile uncovered clear activation of EMT including reprogramming of fibroblasts to migratory cells exhibiting markers of myofibroblasts, along with the upregulation of MMPs and suppression of apical junction genes, likely triggered by TGF $\beta$ signaling activation. Further investigations to determine the cellular origins of EFE fibroblasts are essential to delineate EFE pathogenesis. Moreover, the role of cilia signaling pathways that govern Epi-MCs expansion needs to be fully addressed.

Finally, consistent with the previous transcriptomic analysis of $A L M S 1$ mutants in other species [37, 38], RNA-seq data derived from pEFE fibroblasts revealed induced exon guidance and increased secretory activity, endosomal transport and cellular trafficking. Furthermore, retina, leptin and insulin-related pathways were dysregulated [Supplemental Table 6]. Since obesity, insulin resistance and related metabolic defects are cardinal characteristics of AS [39], it is quite interesting to observe that the insights gained from transcriptome analysis of pEFE fibroblasts recapitulate physiological defects of AS patients and mouse AS models. These conserved effects underscore the utility of integrated genomics analysis (Exome/transcriptome) in patient-derived cells to interpret human phenotypes.

Several limitations need to be acknowledged in our study. Our functional studies are limited to in vitro analysis. Further mechanistic studies of Alms1 mediated ciliary signaling in Alms1 mouse models are nessecary to fully elucidate the mechanisms that underlie pEFE fibrosis and neonatal cardiomyocyte proliferation in AS. It would be essential to characterize ALMS1 function and potential downstream mediators that might impact neonatal heart maturation and to identify disease-specific therapeutic targets.

\section{Methods}

Human Studies: All human studies were conducted in accordance with regulation of the University of California Los Angeles (UCLA) Institutional Review Board (IRB). Subjects provided written informed consent to participate in this study. Electronic medical record, family pedigree and specimen collection were acquired through the UCLA Congenital Heart Defect (CHD) BioCore [21] following the UCLA-IRB approved protocols. Specimens were de-identified and coded following acquisition. pEFE diagnosis was determined based on clinical pathological examination of the explanted heart.

Skin and Muscle Biopsy, Heart Tissue Specimens: Using betadine, then alcohol wipe, the skin was prepped and draped in a sterile fashion. For local anesthesia, approximately $1 \mathrm{ml}$ Lidocaine $1 \%$ was injected intradermally and $3 \mathrm{~mm}$ diameter biopsy punch was performed through the skin. The biopsy sample was placed in DMEM culture media and transported to the lab for adherent primary dermal fibroblast culture following standard protocol. The control human neonatal dermal fibroblasts (hNDFs) were purchased from ATCC. Heart tissue sections were obtained from UCLA clinical pathology laboratory. All samples were de-identified and coded upon procurement.

Whole Exome Sequencing (WES): Genomic DNA was extracted using standard methods (Purelink Genomic DNA Mini Kit, Invitrogen) at the UCLA Congenital Heart Defect-BioCore. Library preparation, 
sequencing and data analysis were performed at the CCRD (California Center for Rare Disease) and the UCLA Clinical Genomics Center, using the CLIA (Clinical Laboratory Improvement Amendments) and CAP (College of American Pathologists) -validated protocols. Genomic DNA (3 $\mu \mathrm{g})$ from the proband and parents was subjected to library preparation and exome capture following the Agilent SureSelect Human All Exon 50 Mb Illumina Paired-End Sequencing Library Prep Protocol. Sequencing was performed on an Illumina HiSeq2000 as a 50 bp paired-end run. For each sample, approximately 200 million independent paired reads were generated for an average coverage of 140X of RefSeq protein coding exons and flanking introns ( +2 bp) with at least $95 \%$ of these bases covered at $\geq 10 X$. The sequences were aligned to the hg19/b37 genome release using Novoalign. PCR duplicates were marked using Picard. Genome Analysis Toolkit (GATK) [40] was used for indel realignment and base quality recalibration. Both SNVs (single nucleotide variants) and small INDELs (insertions and deletions) were called using GATK unified genotyper. All variants were annotated using the customized VEP (variant effect predictor) engine from Ensembl. Regions of homozygosity by descent were determined using PLINK. Rare protein-altering variants were examined under different inheritance models. Rare variants with minor allele frequency $<1 \%$ in public databases were retained for analysis. Variants were classified based on their pattern of inheritance, predicted consequence at the protein level, and their location within the gene. All variants were interpreted in the context of the patient's phenotype.

RNA Sequencing and Bioinformatics Analysis: RNA sequencing, data processing, and bioinformatics analysis steps follow exactly the procedures we previously described $[23,24]$. Briefly, total RNAs were isolated, ribosomal RNAs were depleted (RiboZero Gold, Illumina), and the resulting RNA transcripts were processed into short fragments (about 200 500nt). Strand-specific cDNA libraries were constructed using random hexamer-primed reverse transcription and subsequently were used to generate secondstrand. Sequencing adaptors were ligated using the Illumina Paired-End Sample Prep Kit. Fragments of 200 bp were isolated by gel electrophoresis to select the suitable fragments for PCR amplification. Agilent 2100 Bioanalyzer and ABI StepOnePlus Real-Time PCR System were used for quantification and evaluation of the library quality. Lastly, the libraries were sequenced according to Illumina HiSeqTM 4000 sequencer protocol (BGI-Genomics) in paired-end sequencing mode (2 x $100 \mathrm{nt}$ long reads). After adaptors removal and quality control procedures, reads containing ribosomal RNA (rRNA) were removed by SOAP (Short Oligonucleotide Alignment Program) alignment. Subsequently the raw reads were aligned to reference genome using Tophat. The background noise was reduced by employing fragments abundance threshold and coverage analysis considering only those of 100 bp or longer. HISAT2 (Hierarchical Indexing for Spliced Alignment of Transcripts) was used for mapping step and transcript assembly. Normalized gene expression values were presented using mean reads per kilobase of transcript per million mapped reads (RPKM).

Differential Gene Expression (DGE) Analysis: DGE analysis was performed with expression levels normalized for gene length, library size and GC contents. DEseq2 algorithms was used to assess differential expression in log2 [normalized RPKM] values for each gene. Significant results were reported at Benjamini_Hochberg FDR $\leq 0.05$. The statistical significance for DGE was assessed by Fisher's exact test. Pearson's correlation coefficients ( $r$ ) for gene expression were calculated in R. Principal Component 
Analysis (PCA) was conducted using R function prcomp. The top 1000 varied mRNAs based on alignment results were used to generate PCAs. The heat map function of R, which employs a hierarchical cluster algorithm, was used to draw heat map figure. The log2-transformed data were preprocessed by median centering of the data for each set and then hierarchically clustered using centered correlation as the similarity metric and average linkage as the clustering method. Volcano plot function of R was used to visualize upregulated and downregulated genes.

Other Bioinformatics and Computational Methods: Expression data analysis was supported by functional enrichment analysis, including gene ontology analysis using the Gene Set Enrichment Analysis (GSEA) suite, which calculates the global significance scores by summarizing the overall level of statistical significance of each gene in each pathway. Pathway analysis of the differentially expressed genes was done using Kyoto Encyclopedia of Genes and Genomes (KEGG) Pathways. For upstream analysis, QIAGEN's Ingenuity Pathway Analysis (IPA) was used.

Quantitative Real-Time PCR: Total RNA was isolated from cells or tissue using an RNeasy Mini Kit (QIAGEN). For reverse transcription, one microgram of total RNA was used to generate first-strand cDNA with random primers (Applied Biosystems). Real-time PCR was performed using the SYBR Green Mix (BioRad) on the CFX96 Real-time System (Bio-Rad). Data were presented as average \pm SEM. Two-tailed Student's $t$ tests were used to calculate statistical significance.

Histology and Immunofluorescence Staining: Cells were fixed in 4\% (v/v) formaldehyde in PBS for 20 minutes. After rinsing with PBS, the cells were incubated with $0.1 \%$ Triton X-100 in PBS for 15 minutes at room temperature and rinsed. Heart tissue was fixed in $4 \%(\mathrm{v} / \mathrm{v})$ formaldehyde, embedded into paraffin, and cut into 5- $\mu \mathrm{m}$-thick tissue sections. After deparaffinization, slides were subjected to antigen retrieval. After blocking in PBS containing 10\% bovine serum albumin for 1 hour, the cells or tissue sections were incubated with primary antibodies overnight and then appropriate AlexaFluor-conjugated secondary antibodies (Invitrogen) for 1 hour. Cell nuclei were eventually counterstained by DAPI in the mounting medium (Invitrogen). Images were recorded and analyzed on a Revolve microscope (ECHO). Primary antibodies used to detect the expression of the proteins of interest are ALMS1 (Abcam), Histone H3 (Cell Signaling Technology), Phospho-Histone H3 (Millipore), Ac-alpha-Tubulin (Cell Signaling Technology), Collagen IV (Cell signaling Technology) and Pericentrin (Abcam).

Cell Culture and Small Interfering RNA Studies: Neonatal rat ventricular myocytes (NRVMs) and neonatal rat cardiac fibroblasts (NRCFs) were isolated from 2-day/old rat pups and cultured in DMEM/10\% (vol/vol) FBS with antibiotics at $37^{\circ} \mathrm{C}$ and $5 \% \mathrm{CO} 2$. pEFE patient-derived dermal fibroblasts and human neonatal dermal fibroblasts (hNDFs) were cultured in fibroblast growth medium (ATCC). Transient transfection of cells with siRNA (Bioland Scientific) was performed in 6-well plates using RNAiMAX (Invitrogen). Rat Alms1-siRNA (30 pmol/well) was transfected into NRVM or NRCF cultured in DMEM. For hNDFs, human ALMS1-siRNA (30pmol/well) was used. After $48 \mathrm{~h}$ or $72 \mathrm{~h}$ of incubation, the cells were harvested. 
Proliferation Assay: An xCELLigence Real Time Cell Analysis (RTCA) Single Plate (SP) instrument (Agilent) was used to monitor real-time cell proliferation. This technology works by measuring electron flow transmitted between gold microelectrodes fused to the bottom surface of a 96-well RTCA E-plate, in the presence of an electrically conductive solution such as tissue culture medium. Adhering cells disrupt the interaction between the electrodes and the solution and thus impede electron flow. The impedance of electron flow caused by adherent cells is calculated via computed mathematical algorithms and reported as an arbitrary parameter called Cell Index $(\mathrm{Cl})$, the magnitude of which reflects the rate and extent of cell proliferation. Briefly, patient-derived dermal fibroblasts and control hNDFs were cultured in fibroblast growth medium (ATCC) and seeded at a density of 2500 cells per well in a final volume of $100 \mu$ into a 96-well RTCA E-plate. The E-plate was placed in the RTCA SP station housed inside a standard CO2 cell culture incubator. Cellular impedance data ( $\mathrm{Cl}$ value) was continuously recorded over a 4-day period using the xCELLigence RTCA Software installed on the control unit outside the incubator. Subsequently $\mathrm{Cl}$ curves were analyzed and the Slope parameters were plotted to determine cell proliferation activity over time. Data were presented as average +SEM. A two-tailed Student's $t$-test was used to calculate statistical significance.

Migration Assay: An xCELLigence RTCA Double Purpose (DP) instrument (Agilent) was used to monitor real-time cell migration. This instrument utilizes an electronically integrated Boyden chamber, the CIM plate, to make continuous measurements of electrical impedance generated by cell migration through the microporous membrane of the upper chamber to the gold microelectrodes-fused bottom surface of the lower chamber. Briefly, patient-derived dermal fibroblasts and control hNDFs, or Alms1 siRNA treated neonatal rat cardiac fibroblasts (NRCFs) and scramble treated NRCFs, or ALMS1 siRNA treated hNDFs and scramble treated hNDFs, were cultured in DMEM with $10 \%$ FBS and passaged the day prior to the experiment. Cells in the range of $60-80 \%$ confluence were trypsinized using $0.05 \% /$ Trypsin/EDTA solution, washed once with serum-free medium (SFM), and resuspended in SFM. Subsequently cells were seeded at a density of $10,000 / 20,000$ cells per well in a final volume of $100 \mu$ into the top chamber of a CIMPlate16 (10,000 cells per well for human dermal fibroblasts; 20,000 cells per well for NRCFs). The CIM plate containing the cells were placed at room temperature for 30 minutes to allow the cells to settle down to the bottom surface (the microporous membrane) of the upper chamber, and then loaded into the DP System inside the incubator. Impedance signal was measured with an interval of 15 minutes for 24 hours. Subsequently $\mathrm{Cl}$ curves were analyzed and the Slope parameters were plotted to determine cell migration activity. Data were presented as average +SEM. A two-tailed Student's t-test was used to calculate statistical significance.

FACS Analysis: pEFE patient-derived dermal fibroblasts and control hNDFs were cultured in DMEM with $10 \%$ FBS to $40 \%-50 \%$ confluence, followed by cell cycle synchronization in serum-free DMEM for 16 hours. The cells were subsequently treated with 10 $\mu \mathrm{M}$ Edu (5-ethennyl-2-deoxyuridine) in DMEM with $10 \%$ FBS for 24 hours, incubated in DMEM with 10\%FBS for another 24 hours, followed by fixation with $4 \%$ paraformaldehyde (PFA). The cells were then subjected to FACS analysis. 
Statistics: Quantified results are presented as mean \pm SEM. Student's $t$ test (unpaired, 2-tailed) and ANOVA with post-hoc Kruskal-Wallis were used for comparing 2 groups and more than 2 groups, respectively; $P$ value less than or equal to 0.05 was considered significant, unless specified otherwise. The correlation of gene expression for each mRNA/trait pair was calculated using Pearson's correlation and Benjamini-Hochberg correction methods. A Benjamini-Hochberg-adjusted correlation $P$ value less than or equal to 0.05 was considered significant.

\section{Declarations}

Ethics Approval and Consent to Participate: All human studies were conducted in accordance with the regulation of the University of California Los Angeles Institutional Review Board (IRB). All subjects provided written informed consent to participate in this study. Electronic medical records, 3-generation family pedigree and specimen collection were acquired through the UCLA Congenital Heart Defect (CHD) BioCore following the UCLA-IRB approved protocols. All specimens were de-identified following acquisition.

Consent for Publication: Informed consent for participation in research study, reporting and publication was obtained for all subjects upon enrollment according to the UCLA-IRB approved protocol.

Availability of Data and Materials: The datasets used and analyzed during the current study are available from the corresponding author upon reasonable request. Upon acceptance of this manuscript, WES and RNA-seq datasets will be deposited in the NCBI's Gene Expression Omnibus repository under Neonatal Heart Maturation SuperSeries GSE85728 (http://www.ncbi.nlm.nih.gov/geo/query/acc.cgi? acc=GSE85728/) or according to the journal instruction.

Competing Interests: The authors declare that they have no competing interests.

Funding: This work was supported by grants from NIH/NHLBI 1R01 HL153853-01 (MT), the American Heart Association Career Development Award 18CDA34110414 (MT), the Department of DefenseCongressionally Directed Medical Research Programs W81XWH-18-1-0164 (MT), and the David Geffen School of Medicine Cardiovascular Theme Research Innovation Seed Grant, and the UCLA Center for Clinical Translational Science Institute Research Grant NIH/UL1TR000124 (MT), Unrestricted grant to the Department of Ophthalmology at the USC Keck School of Medicine from Research to Prevent Blindness, New York, NY (AN) and the Las Madrinas Endowment in Experimental Therapeutics for Ophthalmology (AN).

Authors' Contribution: MT conceived the project, designed and performed the research, analyzed most of the data, obtained funding, and wrote the manuscript. $Y Z$ and $X K, V F$ and $Z M$ performed experiments and participated in data analysis and manuscript writing. LW and AE assisted in bioinformatics analysis. GF, MF and KN performed pathological studies. RB and GVA contributed human specimens. NH, JA, MF, GS and LR participated in patient enrollment and provided clinical insights. SP and AN contributed retina 
evaluation. FQR, RS, WWG and SFN participated in genetic studies. All authors contributed to the manuscript reviewing and editing.

Acknowledgments: We acknowledge the support of the UCLA Children's Discovery and Innovation Institute, California Center for Rare Disease (CCRD) at the UCLA Institute for Precision Health, the Clinical Genomics Center, the UCLA Animal Physiology Core, and the UCLA Congenital Heart Defects BioCore.

\section{Non-standard Abbreviations}

pEFE: Primary Endocardial Fibroelastosis

DCM: Dilated Cardiomyopathy

WES: Whole Exome Sequencing

RNA-seq: RNA sequencing

EMT: Epithelial Mesenchymal Transition

EM: Electron Microscopy

AS: Alstrom Syndrome

NRVM: Neonatal Rat Ventricular Myocyte

NRCF: Neonatal Rat Cardiac Fibroblast

hNDF: Human Neonatal Dermal Fibroblast

\section{References}

1. Soares P, Rocha G, Pissarra S, Soares H, Flôr-de-Lima F, CostaS, et al. Neonatal dilated cardiomyopathy. Rev Port Cardiol. 2017;36:201-14.

2. Rodriguez-Fanjul J, Tubio-Gomez S, Carretero Bellón JM, Bautista-Rodriguez C, Sanchez-de-Toledo J. Neonatal Non-compacted Cardiomyopathy: Predictors of Poor Outcome. Pediatr Cardiol. 2020;41:175-80.

3. Kalayinia S, Goodarzynejad H, Maleki M, Mahdieh N. Next generation sequencing applications for cardiovascular disease. Ann Med. 2018;50:91-109.

4. Herkert JC, Abbott KM, Birnie E, Meems-Veldhuis MT, Boven LG, Benjamins M, et al. Toward an effective exome-based genetic testing strategy in pediatric dilated cardiomyopathy. Genet Med. 2018;20:1374-86.

5. Seki A, Patel S, Ashraf S, Perens G, Fishbein MC. Primary endocardial fibroelastosis: an underappreciated cause of cardiomyopathy. Cardiovasc Pathol. 2013;22:345-50. 
6. Sellers FJ, Keith JD, Manning JA. The diagnosis of primary endocardial fibroelastosis. Circulation. 1964;29:49-59.

7. Matsukura H, Miyazaki A, Ichida F, Suzuki Y, Okada T, Nishiya Y, et al. Endocardial fibroelastosis in two siblings with the infantile type and the childhood type. Eur J Pediatr. 1986;145:583-94.

8. Aoki H, Inamura N, Kawazu Y, Nakayama M, Lurie PR. Endocardial fibroelastosis is not a disease. Am J Cardiol. 1988;62:468-70.

9. Fishbein MC, Ferrans VJ, Roberts WC. Histologic and ultrastructural features of primary and secondary endocardial fibroelastosis. Arch Pathol Lab Med. 1977;101:49-54.

10. Westwood M, Harris R, Burn JL, Barson AJ. Heredity in primary endocardial fibroelastosis. Br Heart J. 1975;37:1077-84.

11. Brady AN, Shehata BM, Fernhoff PM. X-linked fetal cardiomyopathy caused by a novel mutation in the TAZ gene. Prenat Diagn. 2006;26:462-5.

12. CollinGB, Marshall JD, Ikeda A, So VW, Russell-Eggitt I, Maffei P, et al. Mutations in ALMS1 cause obesity, type 2 diabetes and neurosensory degeneration in Alström syndrome. Nat Genet. 2002;31:748.

13. Marshall JD, Beck S, Maffei P, Naggert JK. Alstro“m Syndrome. Eur J Hum Genet. 2007;15:1193-202.

14. Alstrom CH, Hallgren B, Nilsson LB, Asander H. Retinal degeneration combined with obesity, diabetes mellitus and neurogenous deafness: a specific syndrome (not hitherto described) distinct from the Laurence-Moon-Bardet-Biedl syndrome: a clinical, endocrinological and genetic examination based on a large pedigree. Acta Psychiatr Neurol Scand Suppl. 1959;129:1-35.

15. Hearn T. ALMS1 and Alström syndrome: a recessive form of metabolic, neurosensory and cardiac deficits. J Mol Med. 2019;97:1-17.

16. Louwa JJ, Corveleyn A, Jia Y, Iqbal S, Boshoff D, Gewillig M, et al. Homozygous loss-of-function mutation in ALMS1 causes the lethal disorder mitogenic cardiomyopathy in two siblings. Eur J Med Genet. 2014;57:532-5.

17. Chang KTE, Taylor GP, Meschino WS, Kantor PF, Cutz E. Mitogenic cardiomyopathy: A lethal neonatal familial dilated cardiomyopathy characterized by myocyte hyperplasia and proliferation. Hum Pathol. 2010; 41:1002-8.

18. Shenje LT, Andersen P, Halushka MK, Lui C, Fernandez L, Collin GB, et al. Mutations in Alstro"m protein impair terminal differentiation of cardiomyocytes. Nat Commun. 2014;5:3416.

19. Paisey RB, Steeds R, Barrett T, Williams D, Geberhiwot T, Gunay-Aygun M. Alström Syndrome. In: Adam MP, Ardinger HH, Pagon RA, Wallace SE, Bean LJH, Stevens K, Amemiya A, editors. GeneReviews. Seattle (WA): University of Washington, Seattle; 1993-2020.

20. Marshall JD, Muller J, Collin GB, Milan G, Kingsmore SF, Dinwiddie D, et al. Alström Syndrome: Mutation Spectrum of ALMS1. Hum Mutat. 2015;36:660-8.

21. Touma M, Reemtsen B, Halnon N, Alejos J, Finn JP, Nelson SF, et al. A Path to implement Precision Child Health Cardiovascular Medicine. Front Cardiovasc Med. 2017;4:36. 
22. Whitfield M, Thomas M, Bequignon E, Schmitt A, Stouvenel L, Montantin G, et al, Mutations in $D N A H 17$, Eencoding a Sperm-Specific Axonemal Outer Dynein Arm Heavy Chain, Cause Isolated Male Infertility Due to Asthenozoospermia. Am J Hum Genet. 2019;105:198-212.

23. Touma M , Kang X, Gao F, Zhao Y, Cass AA, Biniwale R, et al. Wnt11 regulates cardiac chamber development and disease during perinatal maturation. JCI Insight. 2017;2:e94904.

24. Touma M, Kang X, Zhao Y, Cass AA, Gao F, Biniwale R, et al. Decoding the Long Noncoding RNA During Cardiac Maturation: A Roadmap for Functional Discovery. Circ Cardiovasc Genet. 2016;9:395407.

25. Hearn T, Renforth GL, Spalluto C, Hanley NA, Piper K, Brickwood S, et al. Mutation of ALMS1, a large gene with a tandem repeat encoding 47 amino acids, causes Alström syndrome. Nat Genet. 2002;31:79-83.

26. Knorz VJ, Spalluto C, Lessard M, Purvis TL, Adigun FF, Collin GB, et al. Centriolar Association of ALMS1 and Likely Centrosomal Functions of the ALMS Motif-containing Proteins C10orf90 and KIAA1731. Mol Bio Cell. 2010;2:3617-29.

27. Gene Set Enrichment Analysis https://www.gsea-msigdb.org/gsea/index.jsp.

28. Von Gise A, Pu WT. Endocardial and Epicardial Epithelial to Mesenchymal Transitions in Heart Development and Disease. Circ Res. 2012;110:1628-45.

29. Gorący I, Safranow K, Dawid G, Skonieczna-Żydecka K, Kaczmarczyk M, Gorący J, et al. Common genetic variants of the BMP4, BMPR1A, BMPR1B, and ACVR1 genes, left ventricular mass, and other parameters of the heart in newborns. Genet Test Mol Biomarkers. 2012;16:1309-16.

30. Xu X, Friehs I, Zhong Hu T, Melnychenko I, Tampe B, Alnour F, et al. Endocardial fibroelastosis is caused by aberrant endothelial to mesenchymal transition. Circ Res. 2015;116:857-66.

31. Zhang H, Huang X, Liu K, Tang J, He L, Pu W, et al. Fibroblasts in an endocardial fibroelastosis disease model mainly originate from mesenchymal derivatives of epicardium. Cell Res. 2017;27:1157-77.

32. Villalobos E, Criollo A, Schiattarella GG, Altamirano F, French KM, May HI, et al. Fibroblast Primary Cilia Are Required for Cardiac Fibrosis. Circulation. 2019;139:2342-57.

33. Hearn T, Spalluto C, Phillips VJ, Renforth GL, Copin N, Hanley NA, et al. Subcellular localization of ALMS1 supports involvement of centrosome and basal body dysfunction in the pathogenesis of obesity, insulin resistance, and type 2 diabetes. Diabetes. 2005;54:1581-7.

34. Klena NT, Gibbs BC, Lo CW. Cilia and Ciliopathies in Congenital Heart Disease. Cold Spring Harb Perspect Biol. 2017;9:a028266.

35. Pierpont ME, Brueckner M, Chung WK, Garg V, Lacro RV, McGuire AL, et al. Genetic Basis for Congenital Heart Disease: Revisited: A Scientific Statement From the American Heart Association. Circulation. 2018;138:e653-711.

36. Chaudhry B, Henderson DJ. Cilia, mitochondria, and cardiac development. J Clin Invest. 2019;129:2666-8. 
37. Nesmith JE, Hostelley TL, Leitch CC, Matern MS, Sethna S, McFarland R, et al. Genomic knockout of alms1 in zebrafish recapitulates Alström syndrome and provides insight into metabolic phenotypes. Hum Mol Genet. 2019;28:2212-23.

38. Hostelley TL, Lodh S, Zaghloul NA. Whole organism transcriptome analysis of zebrafish models of Bardet-Biedl Syndrome and Alström Syndrome provides mechanistic insight into shared and divergent phenotypes. BMC Genomics. 2016;17:318.

39. Vaisse C, Reiter JF, Berbari NF. Cilia and Obesity. Cold Spring Harb Perspect Biol. 2017;9:a028217.

40. McKenna A, Hanna M, Bank E, Sivachenko A, Cibulskis K, Kernytsky A, Garimella K, Altshuler D, Gabriel S, Daly M, DePristo MA. The Genome Analysis Toolkit: A MapReduce framework for analyzing next-generation DNA sequencing data. Genome Res. 2010;20(9):1297-303.

\section{Figures}

A

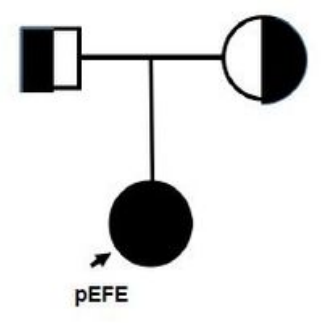

B

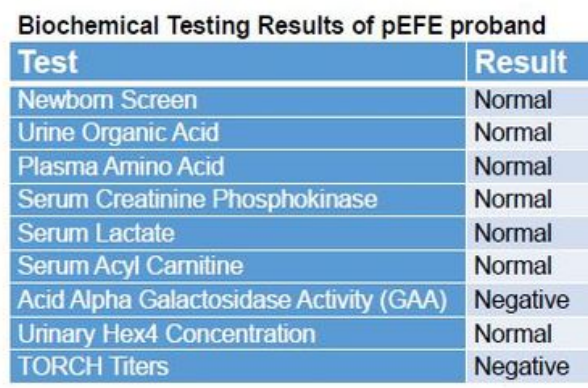

C

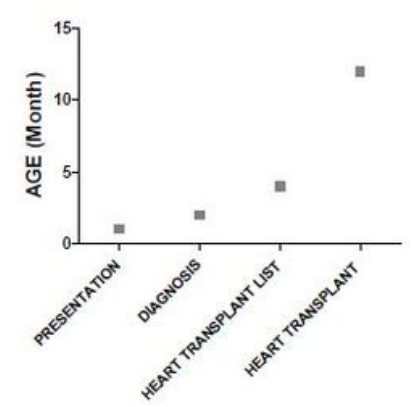

D

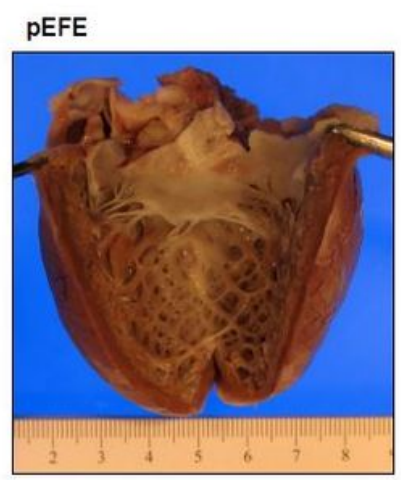

E

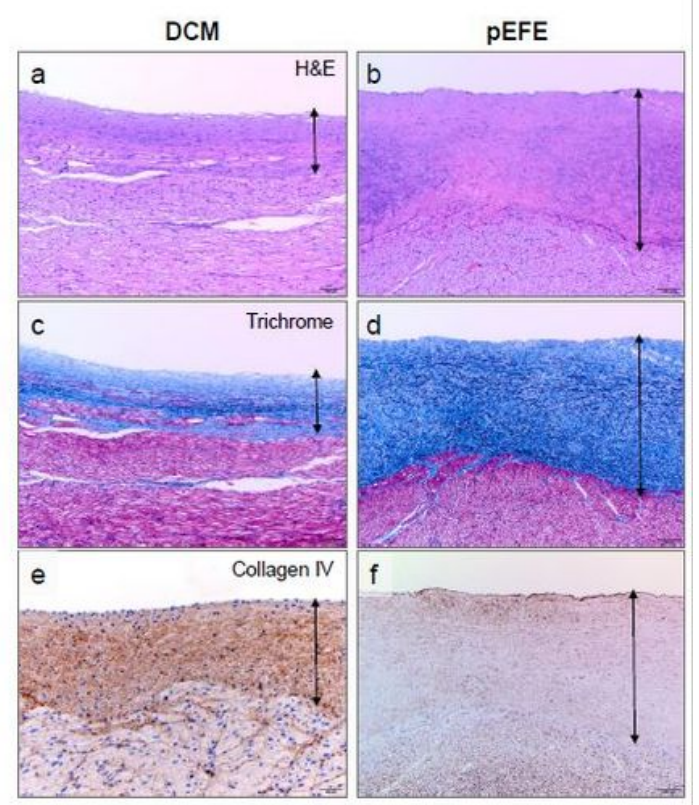

\section{Figure 1}

Primary Endocardial Fibroelastosis (pEFE) is a Distinct Pathological Entity. A. Family pedigree of the pEFE proband affected with novel bi-allelic recessive mutations in three ciliary genes, DNAH8, DNAH17, and ALMS1. B. Table summary of clinical biochemical testing results for the pEFE proband. C. Clinical time course for the pEFE patient. Age at presentation, age at diagnosis, age at listing for heart transplantation, and age at heart transplantation are presented. D. Gross pathology image of pEFE heart demonstrates thickened endocardium. E. Microscopy images of LV sections from a DCM heart (left panel) and a pEFE heart (right panel) demonstrate significant endocardial thickening contributed by deposition of elastic fibrous tissues, while collagen IV was less prominent in pEFE compared to DCM. a 
and b, H\&E histochemistry; $c$ and d, Trichrome histochemistry; e and f, Collagen IV Immunohistochemistry (IHC).

\begin{tabular}{|c|c|c|c|c|c|c|c|c|}
\hline \multicolumn{9}{|c|}{ Rare Protein Altering Homozygous Variants in Cilia-Related Genes Identified in pEFE Proband } \\
\hline Gene & \begin{tabular}{|l|} 
Genomic \\
Position
\end{tabular} & DNA Change & Transcript & Protein Change & Inheritance & Zygosity & MAF & $\begin{array}{l}\text { gmomAD Alt } \\
\text { Allele Freq (AF) }\end{array}$ \\
\hline DNAH17 & $17: 76487553$ & c. $6641 \mathrm{C}>\mathrm{G}$ & NM 173628.3 & p.Ser2214Cys & Maternal/Paternal & Homozygous & 0 & 0 \\
\hline DNAH8 & 6:38840474 & c.7153delT & NM 001206927.1 & p.Phe2385fs & Maternal/Paternal & Homozygous & 0 & 0 \\
\hline ALMS1 & 2:73675594 & c. 1938delA & NM_015120.4 & p.Val647fs & Maternal/Paternal & Homozygous & 0 & 0 \\
\hline
\end{tabular}
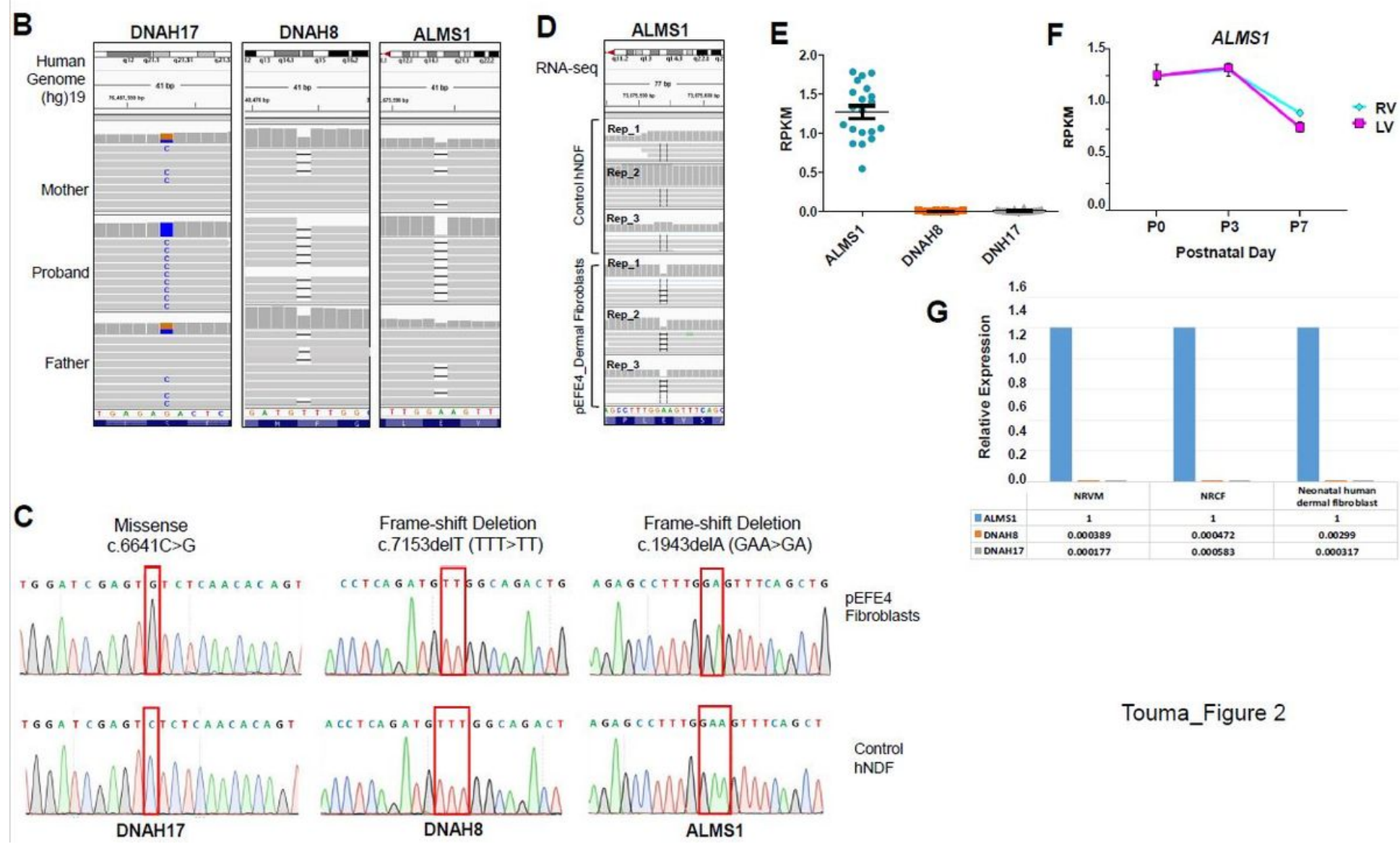

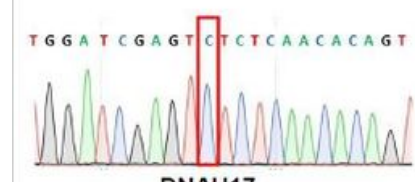

DNAH17
DNAH8

ALMS1

Touma_Figure 2

\section{Figure 2}

Integrated Genomic (WES/RNA-seq) Analysis for Variant Prioritization in pEFE. A. Table Summary of the deleterious bi-allelic variants in cilia-related genes detected in the pEFE proband. B. Integrative genomics viewer windows of Trio WES for the pEFE family show homozygous missense variants in DNAH17, and homozygous deletions in DNAH8 and ALMS1. The parents are heterozygous carriers for each of the variants. C. Sanger sequencing confirms bi-allelic variants in DNAH17, DNAH8 and ALMS1 in pEFE pateint-derived dermal fibroblasts, but not in control human neonatal dermal fibroblasts (hNDFs). D. Integrative genomics viewer window of RNA-seq demonstrates the consequence of ALMS1 variant on ALMS1 RNA reads in pEFE proband-derived fibroblasts compared with control hNDFs. $\mathrm{N}=3$ biological replicates per group. E. RNA-seq derived expression values of ALMS1, DNAH8, and DNAH17 in pEFE dermal fibroblasts. RPKM: Reads per kilo base per million of mapping reads. F. RNA-seq derived expression time course of ALMS1 in neonatal mouse heart right ventricle (RV) and left ventricle (LV) at postnatal day 0 (P0), P3 and P7. G. Expression analysis of of ALMS1, DNAH8 and DNAH17 in neonatal 
rat ventricular myocytes (NRVMs), neonatal rat cardiac fibroblasts (NRCFs) and hNDFs (qRT-PCR). Only ALMS1 was expressed in all three cell types.

A ALMS1 Gene: $2 \mathrm{p} 13.1,23$ exons
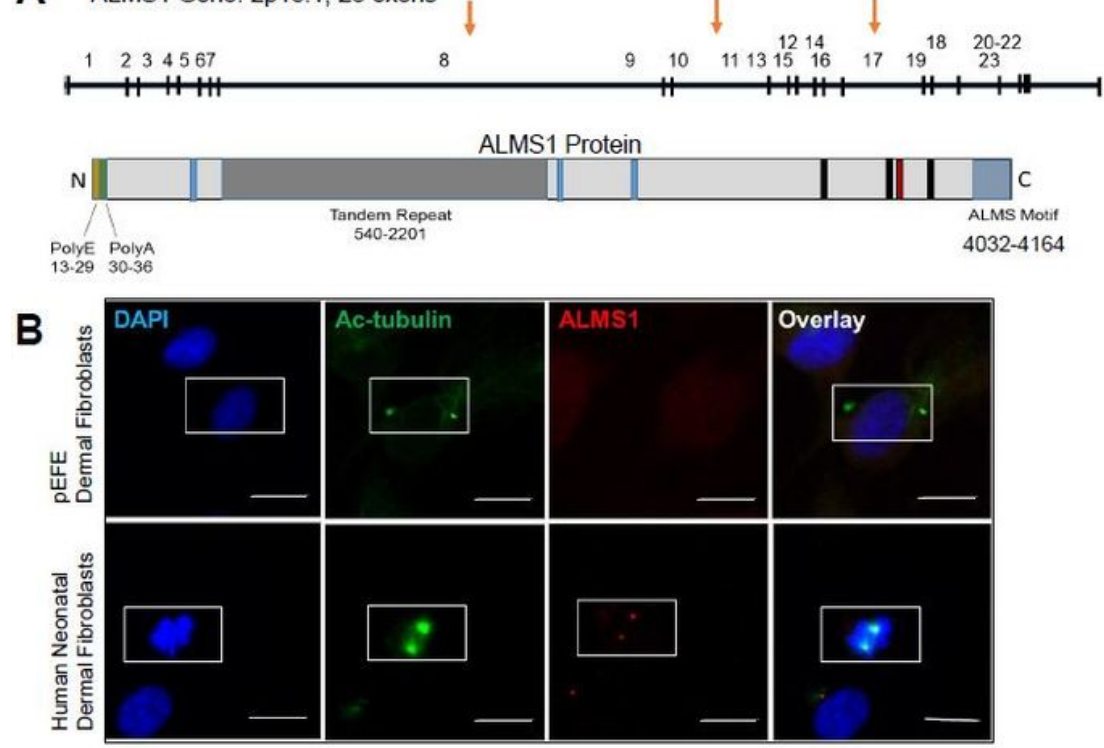

C

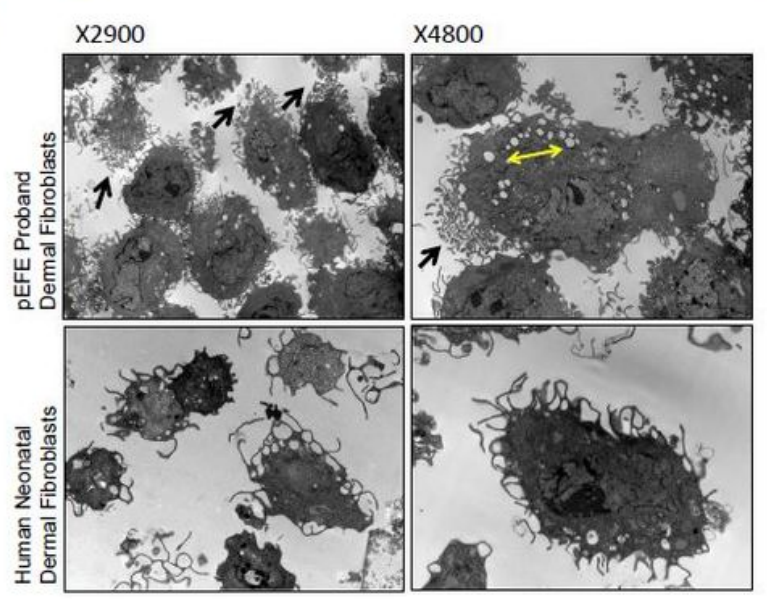

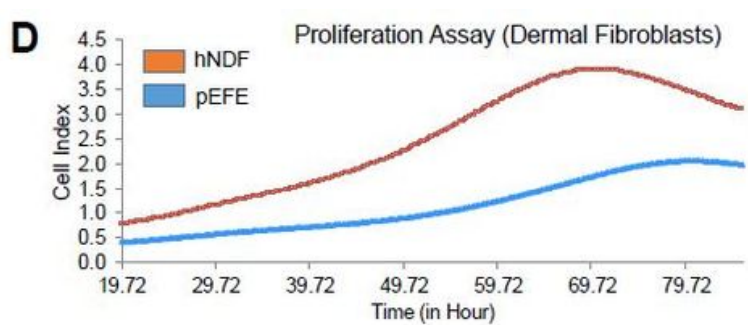
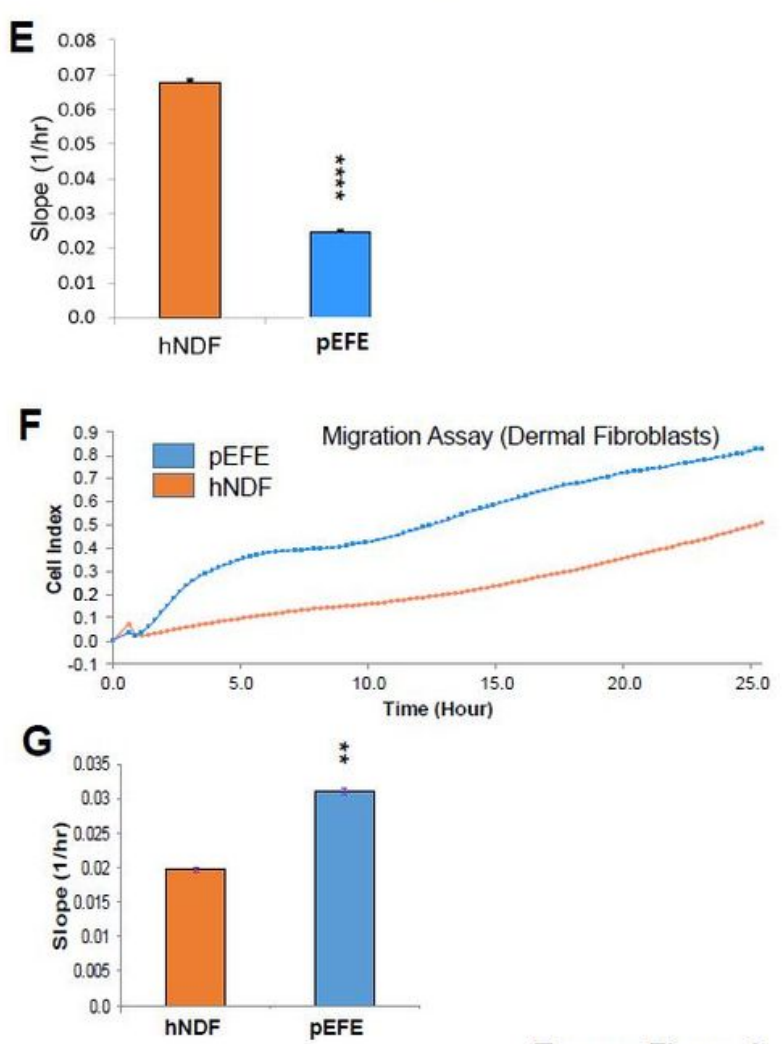

Touma_Figure 3

\section{Figure 3}

The Novel ALMS1 Variant Impedes ALMS1 Protein Expression and Alters the Functional Phenotype of pEFE Fibroblasts. A. Schematic representation of ALMS1 gene (upper) and protein with known structural motifs (lower). B. Representative fluorescence microscopy image of ALMS1 immunocytochemistry (ICC) in pEFE- derived fibroblast (upper panel) and hNDF (lower panel). C. Representative electron microscopy (EM) images of pEFE proband and hNDF fibroblasts show unipolar localization of thin branched microvili was observed in pEFE proband fibroblasts compared to hNDFs (black arrows). Increased intracellular vacuolization was also observed (yellow double headed arrow). D. Proliferation assay of pEFE dermal fibroblasts and control hNDFs. pEFE proband fibroblasts exhibited lower proliferation activity as demonstrated by lower cellular index over time. The proliferation assay was performed using an xCELLigence RTCA SP instrument over an 85-hour period. 2500 cells per well were seeded into the 96-well RTCA E-plate. N=6 biological replicates per group. E. Quantitative analysis of fibroblast proliferation assay shown in D. F. Migration assay of pEFE proband fibroblasts and control hNDFs. pEFE proband 
fibroblasts exhibited enhanced migration activity as demonstrated by higher cellular index over time. The migration assay was performed using an xCELLigence RTCA DP instrument over a 25-hour period. 10000 cells per well were seeded into the upper chamber of CIM-Plate16. $\mathrm{N}=3$ biological replicates per group. $\mathrm{G}$. Quantitative analysis of fibroblast migration assay shown in F.

A

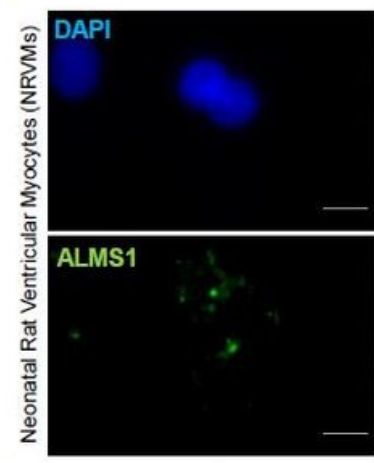

B

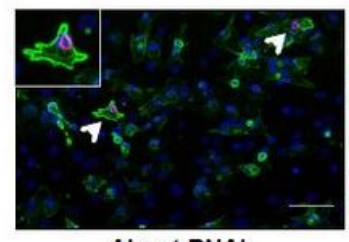

Alms1-RNAi
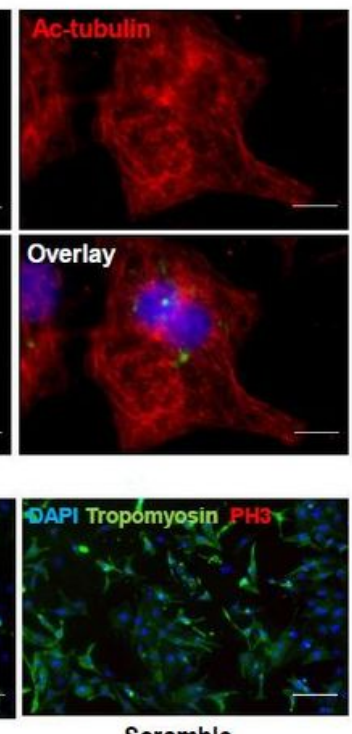

Scramble

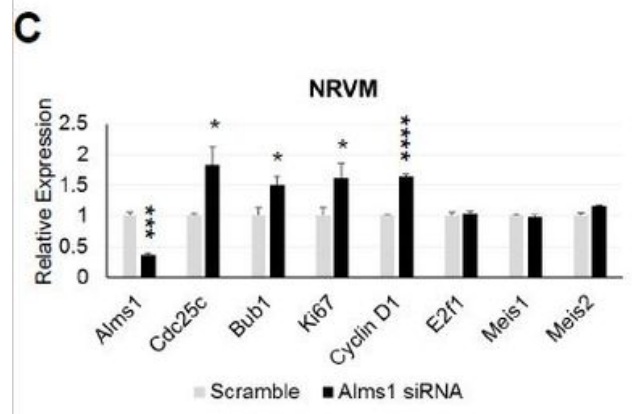

D

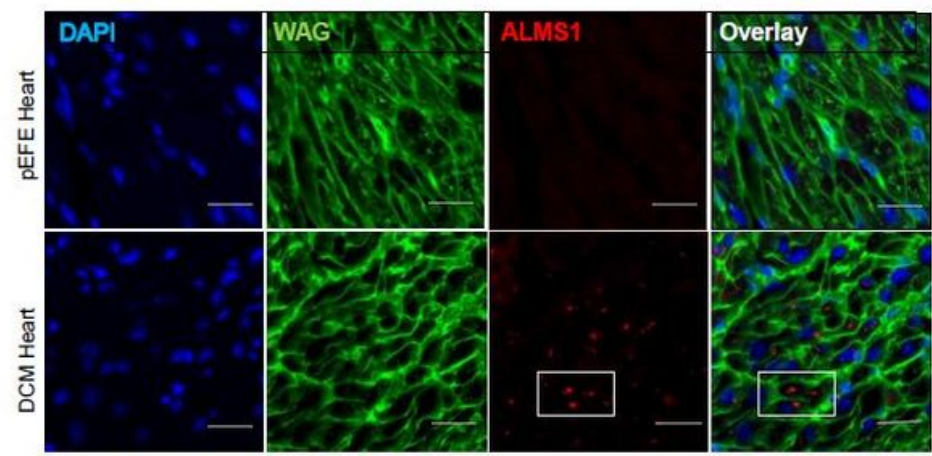

E
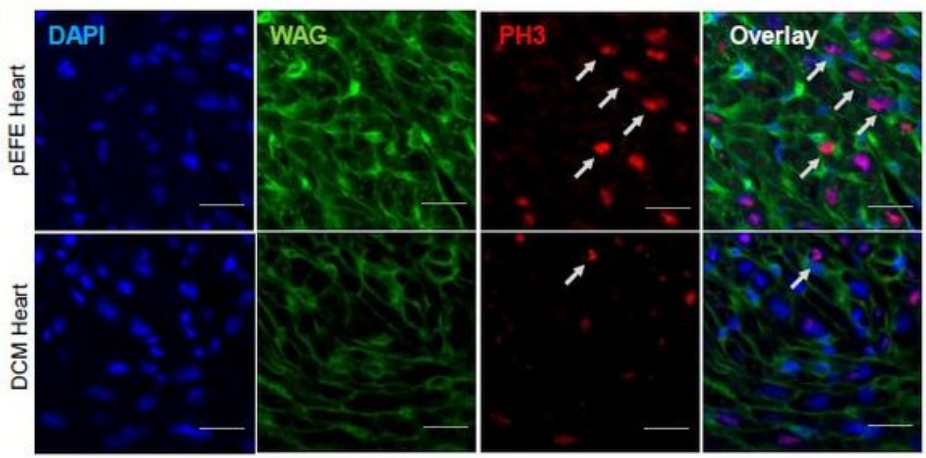

$\mathbf{F}$

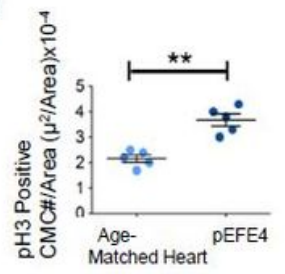

\section{Figure 4}

The Novel ALMS1 Variant Abolishes ALMS1 Protein Expression and Induced Cardiomyocytes Proliferation in pEFE Heart. A. Representative fluorescence microscopy images of neonatal rat ventricular myocytes (NRVMs) show ALMS1 localization at the centromeric poles of the mitotic spindle. B. Representative fluorescence microscopy images of phospho Histone $3(\mathrm{pH} 3)$ stained NRVMs depict enhanced proliferation activity in Alms1- depleted neonatal cardiomyocytes. C. qRT-PCR analysis of

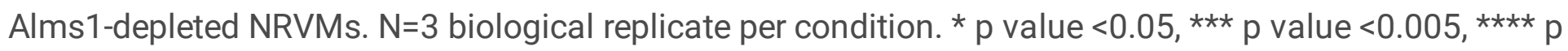
value $<0.001$. D. Representative fluorescence microscopy images of ALMS1 IHC in pEFE heart tissue (middle panel/pEFE) and an age matched proband with DCM (lower panel/DCM). E. Representative fluorescence microscopy images of $\mathrm{pH} 3$-stained heart sections demonstrate increased proliferation activity in pEFE heart (Arrows) compared to age matched DCM heart. F. Quantitative analysis of pH3 positive cardiomyocytes in pEFE heart compared to DCM heart $(n=3$ sections per heart, 5 surface areas per section). 

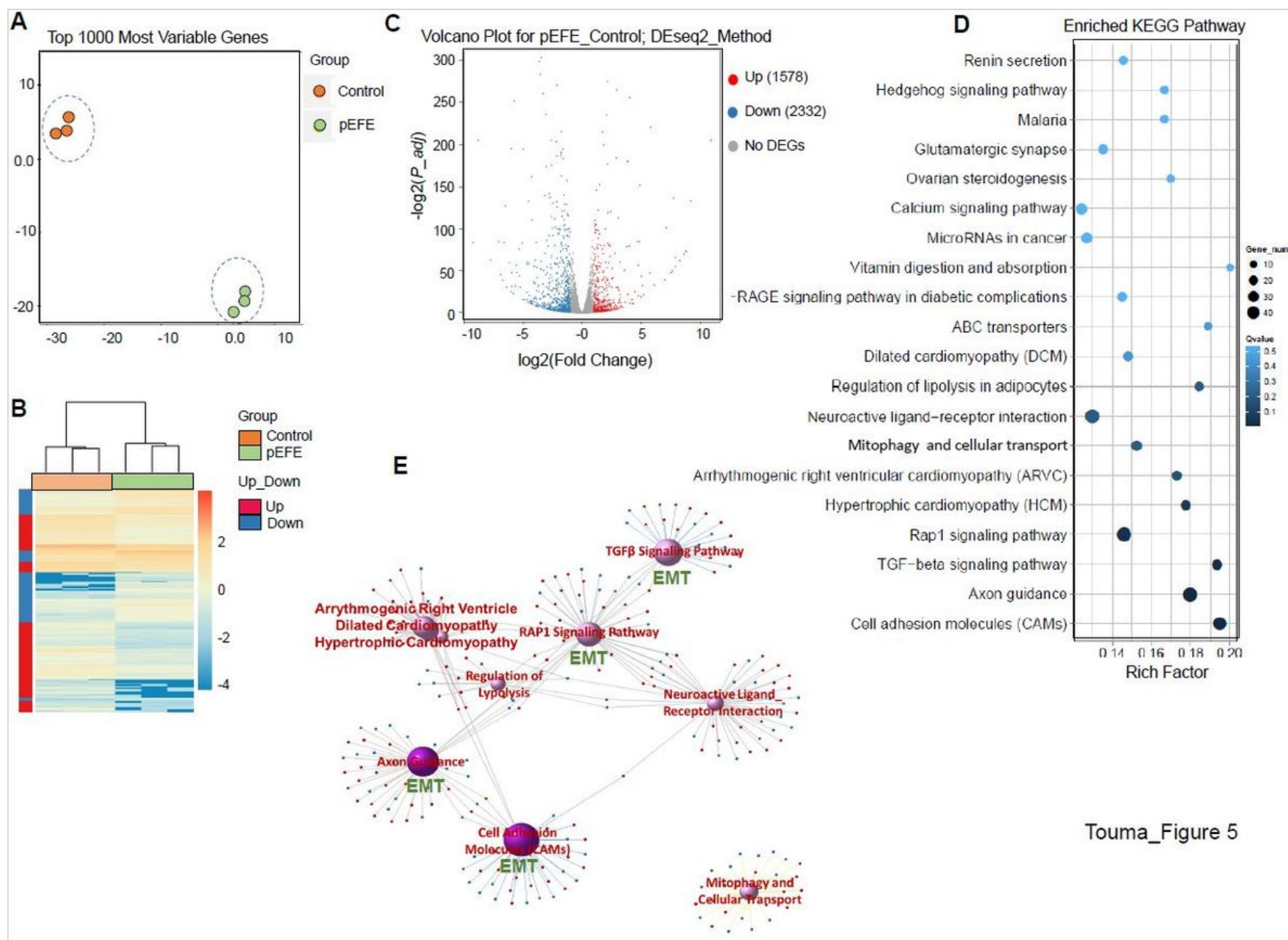

Touma_Figure 5

\section{Figure 5}

The Novel ALMS1 Variant Alters the Molecular Signature of pEFE Fibroblast. A. Principal component analysis of top 1000 varied genes in RNA-seq derived data from pEFE fibroblasts compared to control (hNDF). $\mathrm{N}=3$ biological replicates per group. $\mathrm{B}$. Transcriptome landscape of $\mathrm{pEFE}$ fibroblasts compare to control (hNDFs). $\mathrm{N}=3$ biological replicates per group. $\mathrm{X}$ axis represents the sample. $\mathrm{Y}$ axis represents the differentially expressed genes (DEGs). The color represents the log10 transformed gene expression level. Dark color means high expression level while light color means low expression level. C. Volcano plot of significant DEGs in pEFE fibroblasts vs control (hNDFs) using DEseq2 method. Red: upregulated, Blue: downregulated. D. KEGG pathway analysis of DEGs in pEFE proband fibroblasts vs control (hNDFs). $X$ axis represents enrichment factor. $Y$ axis presents pathway name. The color indicates the q-value (high: white, low: blue). Lower q-value indicates more significant enrichment. Size of the dot indicates DEG number. Rich Factor refers to the value of enrichment factor. Larger value indicates more significant enrichment. E. KEGG-DEGs in pEFE vs control (hNDFs) relationship network. Purple balls represent the top ten enriched pathways. The dark color indicates a significant enrichment ( $Q$ value $<0.01)$, while the light color indicates enrichment that is not significant. Larger ball indicates higher degree of enrichment. The red and blue dots in each network represent the up-regulated and down-regulated genes, respectively. 


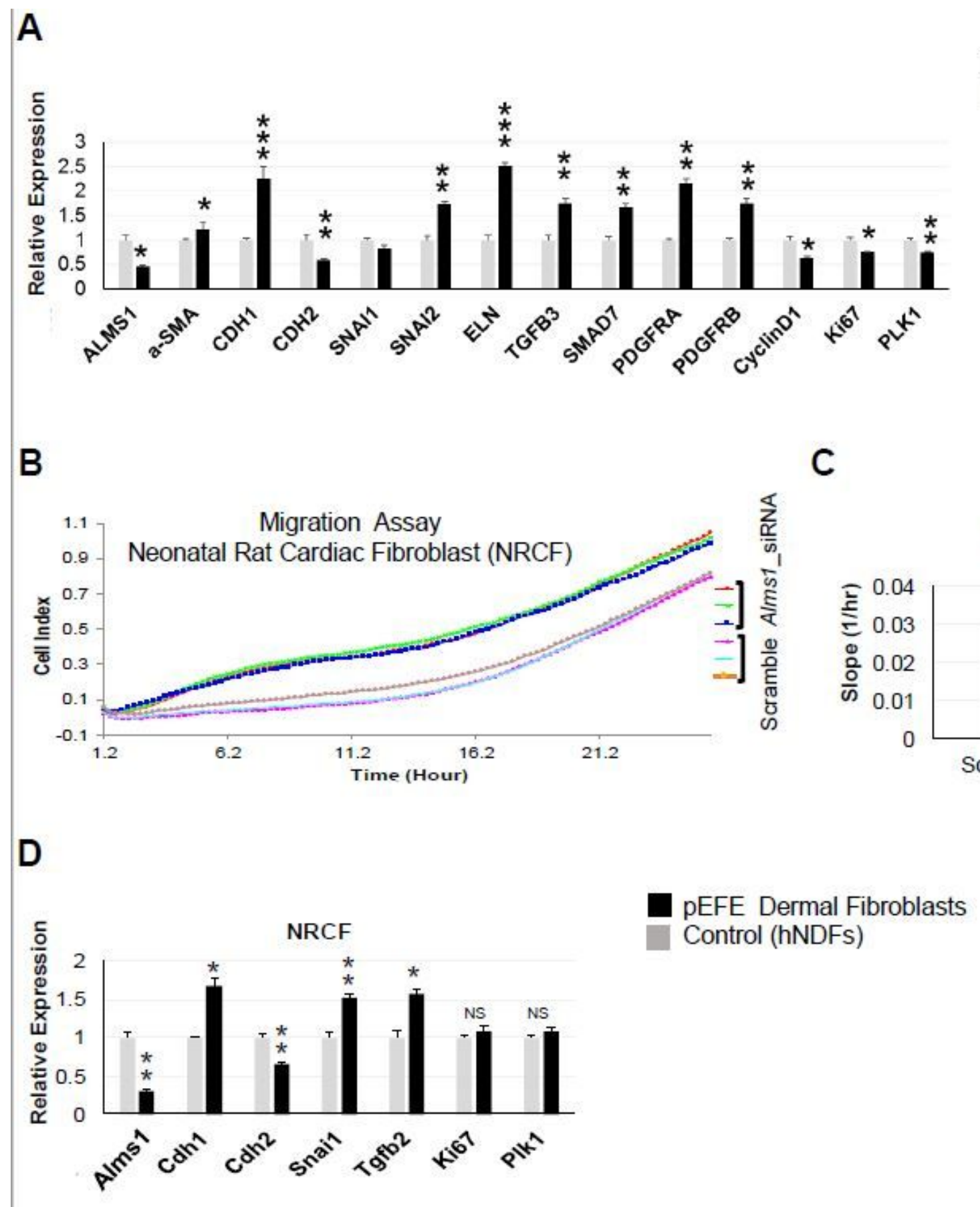

pEFE Dermal Fibroblasts

Control (hNDFs)

B

Touma_Figure 6

Figure 6

ALMS1 Loss Induces Migration and Activates Epithelial to Mesenchymal Transition Program (EMT) in Neonatal Cardiac Fibroblast. A. qRT-PCR analysis of EMT and proliferation marker genes in pEFE proband fibroblasts compared to control (hNDFs). $N=3$ biological replicates per group. ${ }^{*} p$ value $<0.05$, $* \star p$ value $<0.01,{ }^{\star}{ }^{* \star} \mathrm{p}$ value $<0.005$. B. Migration assay of Alms1-depleted NRCFs compared to scramble control. Alms1-depleted NRCFs exhibited enhanced migration activity as demonstrated by higher cellular index 
over time. The migration assay was performed using an xCELLigence RTCA DP instrument over a 25-hour period. 20000 cells per well were seeded into the upper chamber of CIM-Plate16. $\mathrm{N}=3$ biological replicates per condition. C. Quantitative assessment of NRCF migration assay shown in B. ** $p$ value $<0.01$. D. qRT-PCR analysis of EMT and proliferation marker genes in Alms1-depleted NRCF compared to control scramble. Inhibition of Alms1 expression in Alms1 siRNA treated NRCFs was confirmed as well. $\mathrm{N}=3$ biological replicate per condition. * $\mathrm{p}$ value $<0.05, * * \mathrm{p}$ value $<0.01$.

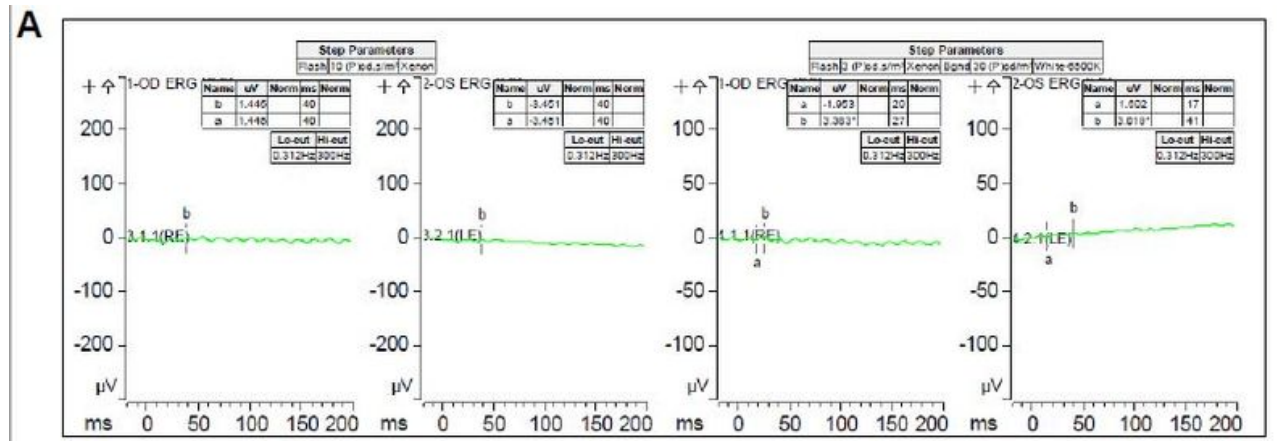

B

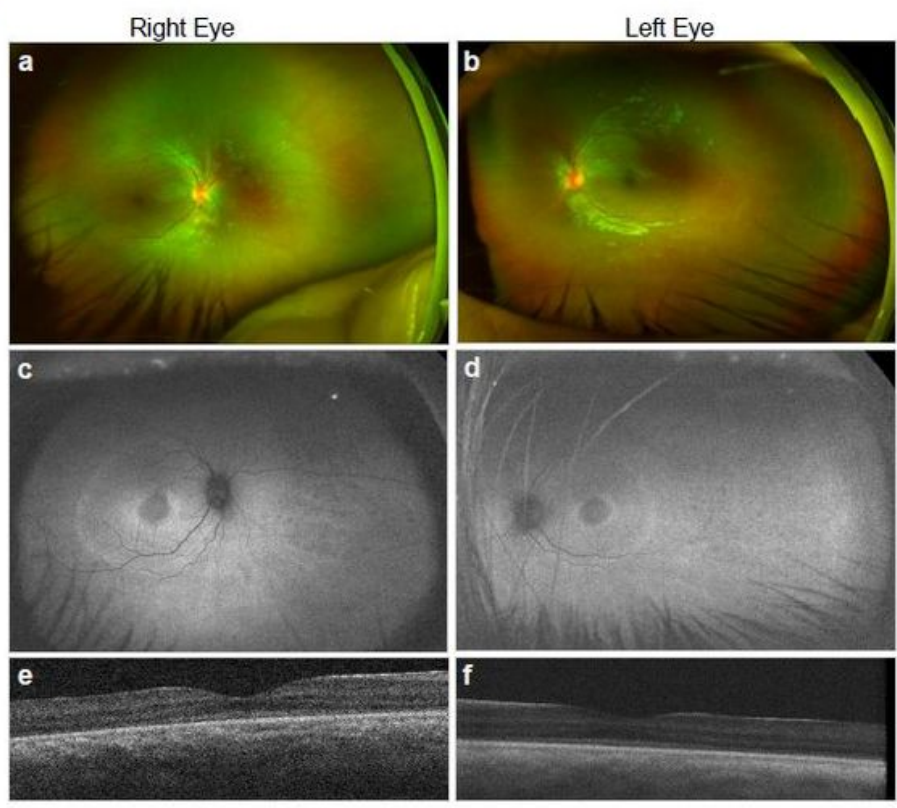

Touma_Figure 8

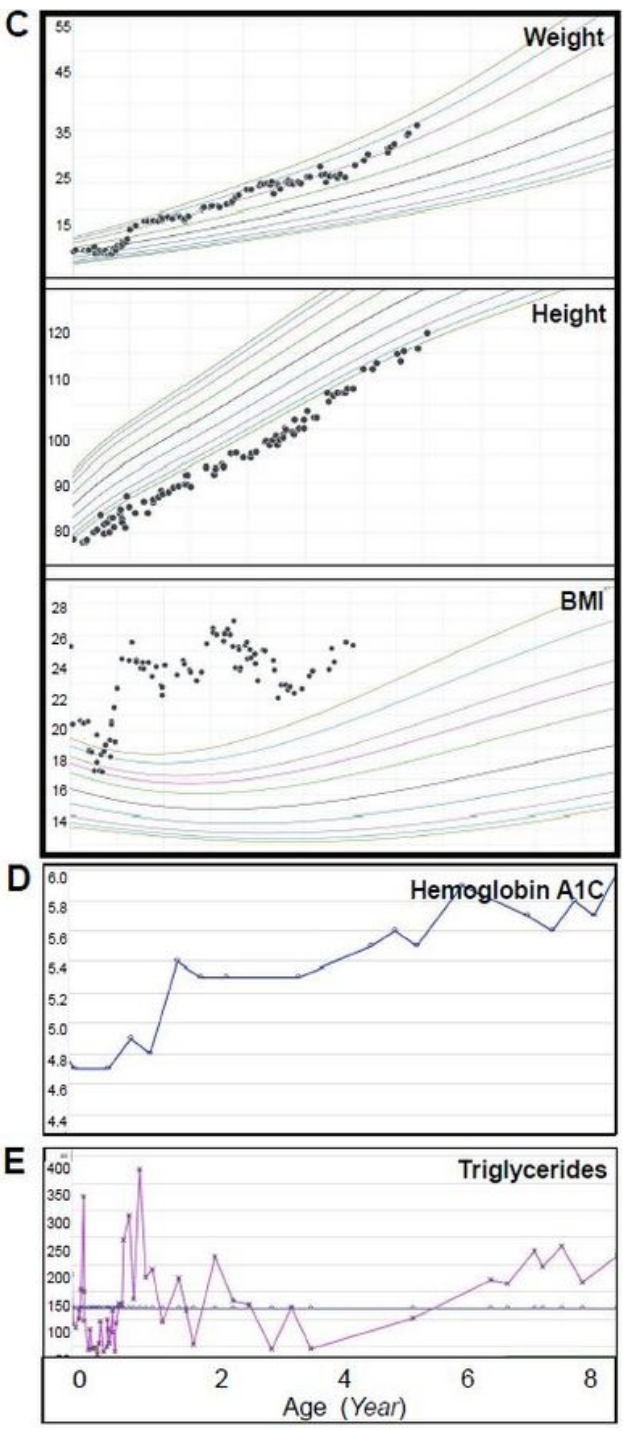

Figure 7

The pEFE Proband Develops other Manifestations of Alstrom Syndrome. A. Electroretinography (ERG) results of the pEFE proband at 7 years of age indicate visual impairment. B. Retinal findings in the $p E F E$ proband. (a-b) Optos widefield pseudocolor fundus images show mild pigmentary changes in the retinal periphery of the right (a) and left (b) eyes. (c-d) Fundus autofluorescence imaging highlights the findings associated with ALMS1-associated retinal dystrophy including central hypoautofluorescence, perifoveal and perimacular hyperautofluorescent rings, and peripheral mottled hyper/hypo autofluorescence in the right (c) and left (d) eyes. (e-f) Spectral-domain optical coherence tomography shows ellipsoid zone attenuation with more pronounced loss in the perifoveal region in the right (e) and left (f) eyes. C. Growth 
charts of the pEFE proband indicate short stature and early onset of obesity (rapid increase of BMI at 2-4 years of age). D. The pEFE proband presents increased Hemoglobin A1C. E. The pEFE proband presents increased serum triglyceride levels.

\section{Supplementary Files}

This is a list of supplementary files associated with this preprint. Click to download.

- SupplementalTables3122021Revised.xlsx

- ToumapEFESupplementalMaterials3122021Revised.pdf 